Annals of Operations Research manuscript No.

(will be inserted by the editor)

\title{
A joint chance-constrained programming approach for the single-item capacitated lot-sizing problem with stochastic demand
}

\author{
Céline Gicquel · Jianqiang Cheng
}

Received: date / Accepted: date

\begin{abstract}
We study the single-item single-resource capacitated lot-sizing problem with stochastic demand. We propose to formulate this stochastic optimization problem as a joint chance-constrained program in which the probability that an inventory shortage occurs during the planning horizon is limited to a maximum acceptable risk level. We investigate the development of a new approximate solution method which can be seen as an extension of the previously published sample approximation approach. The proposed method relies on a Monte Carlo sampling of the random variables representing the demand in all planning periods except the first one. Provided there is no dependence between the demand in the first period and the demand in the later periods, this partial sampling results in the formulation of a chance-constrained program featuring a series of joint chance constraints. Each of these constraints involves a single random variable and defines a feasible set for which a conservative convex approximation can be quite easily built. Contrary to the sample approximation approach, the partial sample approximation leads to the formulation of a deterministic mixed-integer linear problem having the same number of binary variables as the original stochastic problem. Our computational results show that the proposed method is more efficient at finding feasible solutions of the original stochastic problem than the sample approximation method and that these solutions are less costly than the ones provided by the Bonferroni conservative approximation. Moreover, the computation time is significantly shorter than the one needed for the sample approximation method.
\end{abstract}

Keywords Stochastic lot-sizing · Chance-constrained programming · Joint probabilistic constraint · Sample approximation approach · Mixed-integer linear programming

C. Gicquel

Laboratoire de Recherche en Informatique

Université Paris Sud, Paris, France

E-mail: celine.gicquel@lri.fr

J. Cheng

Department of Systems and Industrial Engineering

University of Arizona, Tucson, USA 


\section{Introduction}

The present paper deals with a stochastic optimization problem arising in the context of industrial production planning: the single-item single-resource capacitated lot-sizing problem with stochastic demand.

Lot-sizing can be defined as the clustering of items for manufacturing at the same time. Lot-sizing arises in production whenever setup operations are required in order to prepare the production resource for the processing of a new product. Setup actions can involve many different operations such as cleaning, preheating, tool change, machine calibration or test runs. These operations result in significant costs, the value of which most often does not depend on the quantity produced afterward. To minimize setup costs and obtain a more efficient use of production resources, production should be run using large lot sizes. However, this production policy generates a significant amount of inventory as the production cannot be synchronized with the actual demand pattern. Products will namely have to be held in inventory between the time they are produced and the time they are actually used to satisfy customer demand. This will incur inventory holding costs mainly because of tied up capital. The objective of lot-sizing is thus to provide production plans reaching the best possible trade-off between setup and inventory holding costs while taking into account both the customer demand satisfaction and the technical limitations of the production system.

As can be seen from the above description, one of the key pieces of information needed to make lot-sizing decisions is the timing and level of customer demand. Most existing models and solution algorithms assume that the demand is deterministically known and seek to build the best production plan meeting this demand (see e.g. Jans and Degraeve [11]). However, in practice, the future customer demand often has to be forecasted so that there is a significant degree of uncertainty on its value. Not taking into account forecast uncertainties while planning production may lead to significant organizational and financial difficulties for a manufacturing company. Namely, in case the realized demand is larger than the forecasted one, the company will be faced with stockouts and may have to postpone deliveries or resort to expensive rush production. On the contrary, in case the realized demand is smaller than the forecasted one, the company will be confronted with a costly higher than needed level of inventory.

There is thus a strong industrial need for decision-aid tools to help production managers build production plans where the stochastic nature of the customer demand is explicitly taken into account. The purpose of the present paper is to discuss a mathematical model and a solution algorithm, which could form the basis for such a decision-aid tool.

More precisely, we consider the single-item single-resource capacitated lotsizing problem with stochastic demand. We assume that, even if the demand cannot be deterministically known, a description of the demand uncertainty is available in terms of a probability distribution. We propose to handle this problem through the use of a single-stage stochastic programming approach: we seek to build the production plan before any additional information on the demand realization becomes available and do not consider the possibility of updating it as the demand unfolds over time. We consider the case where the production plan should be feasible for nearly all possible outcomes of the demand. This leads to the formulation of a joint chance-constrained program where the probability that 
an inventory shortage occurs during the planning horizon is limited to a maximum acceptable risk level.

As the resulting probabilistic mixed-integer program is computationally difficult to handle, we consider an approximate solution approach based on the sample approximation approach proposed by Luedtke and Ahmed in [14]. Our main contribution consists in the introduction of a new extension of this approach which we called the "partial sample approximation approach". Similarly to the sample approximation approach, the proposed method relies on a Monte Carlo sampling of the random variables $D_{t}, t=1 \ldots T$, representing the demand in periods 1 to $T$. However, this sampling is carried out on only part of the random variables, more precisely on all random variables except $D_{1}$. Provided there is no dependence between $D_{1}$ and the demand in the later periods, this partial sampling results in the formulation of a chance-constrained program featuring a series of joint chance constraints. Each of these constraints involves a single random variable and defines a feasible set for which a conservative convex approximation can be quite easily built. The main advantage of the proposed method is that, unlike the sample approximation approach, it leads to the formulation of a deterministic mixed-integer linear program (MILP) having the same number of binary variables as the initial stochastic problem. As will be shown by our computational experiments, the proposed method is more efficient at finding feasible solutions of the original stochastic problem than the sample approximation method and these solutions are less costly than the ones provided by the Bonferroni conservative approximation. Moreover, the computation time is shorter than the one needed for the sample approximation method.

The remainder of the paper is organized as follows. We provide in Section 2 an overview of the literature on stochastic lot-sizing. We then describe in Section 3 the joint chance-constrained programming formulation used to model the problem under study. We discuss two previously published solution approaches for this problem: the Bonferroni conservative approximation in Section 4 and the sample approximation approach in Section 5. Section 6 is devoted to the presentation of the proposed partial sample approximation approach. Computational results are provided in Section 7.

\section{Literature review}

Deterministic lot-sizing problems have been extensively studied since the seminal work of Wagner and Whitin [25]. We refer the reader to the literature reviews provided by Jans and Degraeve [11] and by Buschkühl et al [4] for a general introduction to this field and focus in what follows on the lot-sizing problem with stochastic demand. We do not seek to provide an exhaustive literature review but rather aim at giving a general overview of the modeling approaches that have been proposed for this problem.

We propose a classification based on the way in which the consequences of the uncertainty are dealt with in the model. Namely, as the demand is stochastic, we cannot ensure that, for any possible realization of the demand, the computed production plan will be capable of satisfying all the demand so that stockouts may occur during the planning horizon. Several modeling alternatives can be considered to account for this in the optimization problem. 
A first alternative corresponds to single-stage stochastic programming approaches (sometimes referred to as "static uncertainty models" in the lot-sizing literature). In this type of model, the value of all decision variables is decided upon at the beginning of the planning horizon prior to the realization of the uncertain demand. This has some organizational advantages as it fixes the production plan once and for all and allows a good preparation of the production staff members and supply of raw materials. In this type of model, stockouts can be managed using two main different ways. One possibility is to allow backlogging of the demand in case it cannot be satisfied on time but to try to limit its use. This can be done either by penalizing backlogging in the objective function (see e.g. Vargas [24] and Piperagkas et al [20]) or by setting a maximum acceptable level to its expected value (see e.g. Tempelmeier and Herpers [23]). The other possibility is the use of chance constraints imposing that the probability of a stockout stays below an acceptable risk level defined by the production manager. Disjoint chance constraints imposing an upper bound on the probability of a stockout within each planning period have been used e.g. by Bookbinder and Tan [3] and Chen [6]. As mentioned by Tempelmeier in [22], they correspond to defining a minimum value to the period $\alpha$-service level or ready rate often used in supply chain management. Joint chance constraints imposing an upper bound on the probability of a stockout within the whole planning horizon have been used by Beraldi and Ruszczyǹski [1], Küçükyavuz [12] and Zhang et al [26]. They can be understood as a way of defining a minimum value to the horizon $\alpha$-service level.

A second alternative corresponds to multi-stage stochastic programming approaches (also called the "static-dynamic uncertainty models" in the lot-sizing literature). This type of model relies on the fact that only part of the decisions have to be made prior to the realization of the demand and that we may have the possibility to revise the production plan several times during the planning horizon in response to the outcomes of the random demand. This additional flexibility allows a better optimization of the production plan but the resulting nervousness may create practical problems in case of scarce production resources. Two main lines of research can be distinguished here. A first line corresponds to the case where the decisions to be made prior the realization of the demand (first-stage decisions) pertain to the periods where production will occur. Production periods are thus fixed once and for all and the only possible adjustments of the production plan during the planning horizon consists in modifying the production level at the beginning of a production period to take into account the realized demand (see e.g. Bookbinder and Tan [3], Tarim and Kingsman [21] and Tempelmeier [22]). A second line of research considers that the only first-stage decisions to be made are the ones related to the first period of the planning horizon and that all decisions related to the following periods can be decided upon after the demand has realized. This type of model usually relies on a description of the uncertainty through a scenario tree and seeks to define the best possible production policy (see e.g. Di Summa and Wolsey [8], Guan et al [9], Haugen et al [10] and Zhang et al [26]).

The present paper makes use of the first modeling alternative. We namely propose a single-stage stochastic programming approach where all production decisions are made once and for all at the beginning of the planning horizon and we introduce a joint chance-constrained programming formulation to handle the consequences of the uncertainty in the model. We thus deal with the same problem as the one studied in [1] and [12]. Beraldi and Ruszczyǹski [1] assume that the 
demand in each period follows a discrete probability distribution and propose a solution method based on a partial enumeration of the $p$-efficient points of the joint distribution of the random vector representing the cumulative demand over the planning horizon. We recall that a point $v \in \mathbb{R}^{n}$ is a $p$-efficient point of the probability distribution $F$ if $F(v) \geq p$ and there is no point $y \in \mathbb{R}^{n}, y \neq v$ such that $y \leq v$ and $F(y) \geq p$. Küçükyavuz [12] assumes that the demand in each period follows a finite discrete probability distribution and reformulates the joint chance-constrained problem as a mixed-integer linear program. She presents a new class of valid inequalities for this problem which contains the inequalities proposed by Luedtke et al [15] as a special case and shows that these inequalities are numerically efficient in strengthening the mixed-integer linear programming formulation and in reducing the computation time. In the present paper, we also reformulate the problem as a mixed-integer linear problem but we investigate another way of reducing the computation time by relying on a partial sample approximation approach.

\section{Joint chance-constrained programming model}

We consider the single-item single-resource capacitated lot-sizing problem. We first briefly describe the deterministic variant of this problem which can be formulated as a mixed-integer linear program (MILP). We then consider a stochastic variant of this problem where the customer demand to be satisfied is subject to uncertainty and introduce the joint chance-constrained programming formulation studied in the present paper.

\subsection{Deterministic formulation}

We wish to plan production for a single product to be processed on a single capacitated resource over a planning horizon involving $T$ periods indexed by $t=1 \ldots T$.

All problem parameters are assumed to be deterministically known at the time when the production plan is built. $f_{t}$ denotes the fixed setup cost to be paid if production occurs on the resource in period $t, h_{t}$ the inventory holding cost per unit held in stock at the end of period $t$ and $c_{t}$ the production capacity available in period $t . d_{t}$ is the demand to be satisfied at the end of each period $t$ and $d c_{t}=\sum_{\tau=1}^{t} d_{\tau}$ is the cumulative demand over interval [1;t]. The initial inventory level, $I_{0}$, is set to 0 without loss of generality.

We introduce the following decision variables:

$-x_{t}$ : the quantity produced in period $t$.

$-y_{t} \in\{0,1\}:$ the resource setup state in period $t . y_{t}=1$ if a setup occurs in period $t, 0$ otherwise. 
With this notation, the deterministic single-item single-resource capacitated lot-sizing problem can be formulated as follows:

$$
\begin{cases}Z_{D E T}^{*}=\min \sum_{t=1}^{T} f_{t} y_{t}+\sum_{t=1}^{T} h_{t}\left(\sum_{\tau=1}^{t} x_{\tau}-d c_{t}\right) & \\ x_{t} \leq c_{t} y_{t} & \forall t=1 \ldots T \\ \sum_{\tau=1}^{t} x_{\tau} \geq d c_{t} & \forall t=1 \ldots T \\ x_{t} \geq 0 & \forall t=1 \ldots T . \\ y_{t} \in\{0,1\} & \forall t=1 \ldots T .\end{cases}
$$

The objective function (1) corresponds to the minimization of the setup and inventory holding costs over the planning horizon. Note that $\sum_{\tau=1}^{t} x_{\tau}-d c_{t}$ computes the inventory level at the end of period $t$ as the difference between the cumulative production up to $t$ and the cumulative demand up to $t$. Constraints (2) ensure that, if production takes place in period $t$, the corresponding setup costs are incurred and the capacity limit $c_{t}$ is respected. Constraints (3) are the demand satisfaction constraints: they guarantee that the cumulative production over each interval $[1 ; t]$ is large enough to satisfy the cumulative demand over the same interval, and consequently that the inventory level at the end of period $t$, $\sum_{\tau=1}^{t} x_{\tau}-d c_{t}$, is non-negative.

\subsection{Stochastic formulation}

We now consider the case where the customer demand to be satisfied in period $t$ is not perfectly known at the time when the production plan is built. This might be due among others to the fact that the only available information on the future demand is based on forecasts (rather than on firm customer orders) and that forecast inaccuracies are unavoidable. We thus model the demand in period $t$ as a random variable $D_{t}$, the probability distribution of which is assumed to be known. The cumulative demand over periods $1 . . . t$, denoted by $D C_{t}=\sum_{\tau=1}^{t} D_{t}$, is also a random variable. The deterministic parameter $d c_{t}$ is thus replaced by a random variable, $D C_{t}$, in the stochastic formulation of the problem.

This has several implications for the problem formulation. First, replacing $d c_{t}$ by its stochastic counterpart $D C_{t}$ implies that the inventory level at the end of period $t$ is a random variable defined as $S_{t}=\max \left(\sum_{\tau=1}^{t} x_{\tau}-D C_{t}, 0\right)$ (see e.g. [1]). Hence, the value of the inventory holding cost appearing in the objective function, $\sum_{t=1}^{T} h_{t} S_{t}$, is also a random variable. We therefore consider minimizing its expected value $\mathbb{E}\left[\sum_{t=1}^{T} h_{t} S_{t}\right]$ in the stochastic formulation. In the present work, we use the same approximation as the one used by Bookbinder and Tan [3] and Beraldi and Ruszczyǹski $[1]$ to compute $\mathbb{E}\left[\sum_{t=1}^{T} h_{t} S_{t}\right]$, namely: 


$$
\begin{aligned}
\mathbb{E}\left[\sum_{t=1}^{T} h_{t} S_{t}\right] & =\mathbb{E}\left[\sum_{t=1}^{T} h_{t} \max \left(\sum_{\tau=1}^{t} x_{\tau}-D C_{t}, 0\right)\right] \\
& \approx \mathbb{E}\left[\sum_{t=1}^{T} h_{t}\left(\sum_{\tau=1}^{t} x_{\tau}-D C_{t}\right)\right] \\
& \approx \sum_{t=1}^{T} h_{t}\left(\sum_{\tau=1}^{t} x_{\tau}-\mathbb{E}\left[D C_{t}\right]\right)
\end{aligned}
$$

With this approximation, our model does not capture the inventory holding cost exactly: it tends to underestimate it in the periods with a negative ending inventory. Note that Zhang et al [26] study a model in which the inventory holding cost is computed exactly through a set of additional constraints.

Second, the fact that $D C_{t}$ is stochastic implies that it might be very costly (and even impossible depending on the support of the probability distributions) to build a production plan ensuring that the cumulative production is large enough to satisfy every possible realization of the demand. We thus have to consider the eventuality that the demand satisfaction constraints (3) will be violated for some demand realizations. As explained in Section 2, a possible way of handling this situation in the optimization problem consists in limiting the probability of these violations through the use of chance constraints. We define a maximum acceptable risk level $\epsilon$ and impose $1-\epsilon$ as an upper bound either on the probability of a stockout within each planning period or on the probability of a stockout in all periods of the planning horizon.

Imposing that the probability of a stockout within each planning period stays below $1-\epsilon$ leads to the following program featuring $T$ disjoint chance constraints.

$$
\begin{cases}Z_{D C C}^{*}=\min \sum_{t=1}^{T} f_{t} y_{t}+\sum_{t=1}^{T} h_{t}\left(\sum_{\tau=1}^{t} x_{\tau}-\mathbb{E}\left[D C_{t}\right]\right) & \\ x_{t} \leq c_{t} y_{t} & \forall t=1 \ldots T \\ \operatorname{Pr}\left(\sum_{\tau=1}^{t} x_{\tau} \geq D C_{t}\right) \geq 1-\epsilon & \forall t=1 \ldots T \\ x_{t} \geq 0 & \forall t=1 \ldots T . \\ y_{t} \in\{0,1\} & \forall t=1 \ldots T .\end{cases}
$$

Enforcing a minimum value to the probability that all demand satisfaction constraints are simultaneously respected leads to the following joint chance-constrained 
program denoted SLS in what follows.

$$
\begin{cases}Z_{S L S}^{*}=\min \sum_{t=1}^{T} f_{t} y_{t}+\sum_{t=1}^{T} h_{t}\left(\sum_{\tau=1}^{t} x_{\tau}-\mathbb{E}\left[D C_{t}\right]\right) & \\ x_{t} \leq c_{t} y_{t} & \forall t=1 \ldots T \\ \operatorname{Pr}\left(\sum_{\tau=1}^{t} x_{\tau} \geq D C_{t}, \forall t=1 \ldots T\right) \geq 1-\epsilon & \\ x_{t} \geq 0 & \forall t=1 \ldots T . \\ y_{t} \in\{0,1\} & \forall t=1 \ldots T .\end{cases}
$$

\subsection{Small illustrative example}

Before discussing solution approaches for problem SLS in more detail, we introduce a small example in order to better illustrate the difference between the disjoint chance constraints (11), imposing a minimum value to the period $\alpha$-service level in each period $t$, and the joint chance constraint (16) imposing a minimum value to the horizon $\alpha$-service level.

In this small example, we consider a planning horizon of $T=5$ periods. We set $c_{t}=100, h_{t}=1, f_{t}=50$ for all periods $t=1 \ldots T$ and $\epsilon=0.20$. We assume that the random demand $D_{t}$ is described by a set of $N=5$ equiprobable scenarios.

Figure 1 provides the numerical values of the cumulative demand $D C_{t}^{i}$ for each scenario $i$ and each period $t$ and the cumulative production over $1 \ldots t$ of two production plans. The first production plan denoted by $x c^{D C C}$ is a feasible solution of problem (9) - (13). Namely, in each period, there is only a single scenario (scenario 1 in periods 1 to 3 , scenario 2 in periods 4 and 5) in which the cumulative production $x c^{D C C}$ is smaller than the cumulative demand $D C_{t}^{i}$. Hence, the probability of a stockout in each individual period is 0.20 and is thus equal to the maximum acceptable stockout risk $\epsilon$. However, the probability that there is no stockout over the whole planning horizon is only 0.6 as 2 scenarios out of 5 display stockouts with $x c^{D C C}$. This means that $x c^{D C C}$ is not a feasible solution for problem SLS. The second production plan, $x c^{J C C}$, which incurs stockouts only in a single scenario (scenario 1 in periods 1 to 3 ), is the least costly production plan complying with the joint chance constraint (16).

In many cases, problem (9) - (13) can be easily solved by replacing each chance constraint (11) by the deterministic constraint $\sum_{\tau=1}^{t} x_{\tau} \geq F_{D C_{t}}^{-1}(1-\epsilon)$, where $F_{D C_{t}}$ denotes the cumulative probability distribution of $D C_{t}$. However, as explained e.g. by Luedtke and Ahmed [14], mathematical programs involving a joint chance constraint such as (16) are still largely intractable except for a few exceptions. This can be explained by two main reasons. First, checking the feasibility of a given solution requires computing the value of the joint probability through multidimensional integration, which can be very time-consuming. Second, the feasible space defined by a joint chance-constraint is in general not convex. Yet, as mentioned e.g. by Nemirovski and Shapiro [18], we need both efficient computation of the probability and convexity of the solution space to efficiently process chance-constraints. This is why a variety of tractable approximations have been 


\begin{tabular}{|c|c|c|c|c|c|c|}
\hline$t$ & 1 & 2 & 3 & 4 & 5 & \\
\hline$x c^{D C C}$ & 30 & 120 & 120 & 210 & 250 & $Z=480$ \\
\hline$x c^{J C C}$ & 30 & 120 & 120 & 220 & 320 & $Z=560$ \\
\hline$D C^{1}$ & 80 & 160 & 200 & 210 & 250 & \\
\hline$D C^{2}$ & 20 & 60 & 120 & 220 & 320 & \\
\hline$D C^{3}$ & 20 & 55 & 90 & 150 & 200 & \\
\hline$D C^{4}$ & 15 & 60 & 120 & 140 & 150 & \\
\hline$D C^{5}$ & 30 & 80 & 90 & 150 & 170 & \\
\hline
\end{tabular}

Fig. 1 Small illustrative example

proposed for chance-constrained problems. They rely either on conservative convex approximations (see e.g. Bonferroni [2], Rockafellar and Uryasev [17], Nemirovski and Shapiro [19]) or on a discretization of the probability distribution through sampling (see e.g. Calafiore and Campi [5], Luedtke and Ahmed [14]).

In Sections 4 to 6 , we first describe the Bonferroni approximation which provides guaranteed feasible solutions of SLS and requires a limited computational effort but may lead to overly conservative and expensive solutions. We then discuss the sample approximation approach developed by Luedtke and Ahmed [14] before presenting the extension proposed in this work, which we refer to as the "partial sample approximation" approach.

\section{Bonferroni conservative approximation}

Let $E_{t}$ be the random event that there is no stockout at the end of period $t$, i.e. that $\sum_{\tau=1}^{t} x_{\tau} \geq D C_{t}$ and $P\left(E_{t}\right)$ be the probability that this event occurs. The Bonferroni inequality states that:

$$
\operatorname{Pr}\left(\cap_{t=1}^{T} E_{t}\right) \geq 1-\sum_{t=1}^{T}\left[1-P\left(E_{t}\right)\right]
$$

By replacing in (16) the joint probability by its lower bound and adjusting the reliability level appropriately, we can divide the joint chance constraint into $T$ individual chance constraints: $\operatorname{Pr}\left(\sum_{\tau=1}^{t} x_{\tau} \geq D C_{t}\right) \geq 1-\frac{\epsilon}{T}$. This leads to the following deterministic mixed-integer linear program denoted by BON:

$$
\begin{cases}Z_{B O N}^{*}=\min \sum_{t=1}^{T} f_{t} y_{t}+\sum_{t=1}^{T} h_{t}\left(\sum_{\tau=1}^{t} x_{\tau}-\mathbb{E}\left[D C_{t}\right]\right) & \\ x_{t} \leq c_{t} y_{t} & \forall t=1 \ldots T \\ \sum_{\tau=1}^{t} x_{\tau} \geq F_{D C_{t}}^{-1}\left(1-\frac{\epsilon}{T}\right) & \forall t=1 \ldots T \\ x_{t} \geq 0 & \forall t=1 \ldots T . \\ y_{t} \in\{0,1\} & \forall t=1 \ldots T .\end{cases}
$$


$F_{D C_{t}}$ is the cumulative probability distribution of the random variable $D C_{t}=$ $\sum_{\tau=1}^{t} D_{\tau}$. In the numerical experiments to be presented in Section 7 , the value of $F_{D C_{t}}^{-1}\left(1-\frac{\epsilon}{T}\right)$ is computed as follows:

- In case $D_{1}, \ldots, D_{T}$ are independent random variables, each following a uniform distribution on the same interval $[L, U]$, we use the fact that $\frac{D C_{t}-t L}{U-L}$ follows the Irwin-Hall distribution of mean $t$ and standard deviation $\sqrt{\frac{t}{12}}$.

- In case $D_{1}, \ldots, D_{T}$ are independent random variables, each following a normal distribution $\mathcal{N}\left(m_{t}, s_{t}\right)$, we use the fact that $D C_{t}$ follows a normal distribution $\mathcal{N}\left(\sum_{\tau=1}^{t} m_{\tau}, \sqrt{\sum_{\tau=1}^{t} s_{\tau}^{2}}\right)$.

- For other cases, we estimate the value of $F_{D C_{t}}^{-1}\left(1-\frac{\epsilon}{T}\right)$ by generating $N_{b}=$ 100000 scenarios, ordering them in the decreasing order of $D C_{t}^{i}$ and selecting the value in position $\left\lceil\frac{\epsilon}{T} N\right\rceil$.

Problem (20)-(24) is a deterministic problem similar to (1)-(5) which can be solved with a limited computational effort. It provides guaranteed feasible solutions of SLS. However, as will be shown by the computational results provided in Section 7 , most often, these solutions are overly conservative and expensive.

\section{Sample approximation approach}

The sample approximation approach proposed by Luedtke and Ahmed [14] aims at providing approximate solutions for joint chance-constrained programs. The main idea consists in replacing the original continuous probability distribution of the random vector (the cumulative demand vector $D C$ in our case) by an empirical discrete finite probability distribution obtained by Monte Carlo sampling. When randomness appears only on the right-hand side of the constraints (which is the case in problem SLS), this leads to the formulation of a large-size mixed-integer linear program, which can be handled by mixed-integer linear programming techniques.

In this Section, we first present how applying the sample approximation approach to problem SLS leads to the formulation of such a large-size MILP. We then investigate two techniques which can be used to ease its resolution by a standard MILP solver: the strong extended formulation discussed by Luedtke et al [15] for joint probabilistic programs with random right-hand sides and the valid inequalities proposed by Leung et al [13] for the deterministic single-item capacitated lot-sizing problem.

\subsection{First MILP formulation}

Let $D C^{1}, \ldots, D C^{i}, \ldots, D C^{N}$ be a Monte Carlo sample of the random vector $D C$. The $N$ sampled scenarios are independent and identically distributed observations of vector $D C$ so that the probability of each scenario $i$ is considered to be equal to $1 / N$.

The sample approximation approach relies on the idea that, given a production plan $x$, the value of the joint probability involved in constraint (16) can be approximately computed as follows: 


$$
\operatorname{Pr}\left(\sum_{\tau=1}^{t} x_{\tau} \geq D C_{t}, \forall t=1 \ldots T\right) \approx \frac{1}{N} \sum_{i=1}^{N} \mathbb{I}\left(\sum_{\tau=1}^{t} x_{\tau}-D C_{t}^{i} \geq 0 \quad \forall t\right)
$$

where $\mathbb{I}($.$) denotes the indicator function taking the value 1$ when . is true and 0 otherwise.

The idea underlying approximation (25) is the following. We check, for each scenario $i$, whether all demand satisfaction constraints are respected by the production plan $x$. We then count the total number $N_{\text {sat }}$ of scenarios where all demand satisfaction constraints are respected and use the ratio $N_{\text {sat }} / N$ as an estimation of the value of the joint probability. The main advantage of this approximation is that it enables to reformulate problem SLS as a mixed-integer linear program.

This is done by introducing a new set of binary variables: $\alpha^{i} \in\{0,1\} . \alpha^{i}$ is defined by $\alpha^{i}=1$ if at least one demand satisfaction constraint is violated in scenario $i, \alpha^{i}=0$ otherwise.

This leads to the following mixed-integer linear program denoted by SA in the sequel of the paper.

$$
\begin{cases}Z_{S A}^{*}=\min \sum_{t=1}^{T} f_{t} y_{t}+\sum_{t=1}^{T} h_{t}\left(\sum_{\tau=1}^{t} x_{\tau}-\mathbb{E}\left[D C_{t}\right]\right) & \\ x_{t} \leq c_{t} y_{t} & \forall t \\ \sum_{\tau=1}^{t} x_{\tau} \geq D C_{t}^{i}\left(1-\alpha^{i}\right) & \forall t, \forall i=1 \ldots N \\ \sum_{i=1}^{N} \alpha^{i} \leq\lfloor N \epsilon\rfloor & \\ x_{t} \geq 0 & \forall t \\ y_{t} \in\{0,1\} & \forall t \\ \alpha^{i} \in\{0,1\} & \forall i=1 \ldots N\end{cases}
$$

Constraints (28) make sure that, if $\alpha^{i}=0$, the $T$ demand satisfaction constraints corresponding to scenario $i$ are satisfied by the production plan $x$. Constraint (29) is the joint probability constraint: it limits the number of violated scenarios to $\lfloor N \epsilon\rfloor$, thus ensuring that the ratio $N_{\text {sat }} / N$ is above $1-\epsilon$.

Problem SA is based on an approximate representation of constraint (16) of problem SLS. As a consequence, there is no definite guarantee that solving problem SA will provide a feasible solution of problem SLS. However, Luedtke and Ahmed [14] showed that, when the uncertainty is located only on the right hand side of the constraints as it is the case here, the probability that problem SA provides a feasible solution to problem SLS increases exponentially fast with the sample size $N$ and tends to 1 when $N$ tends to infinity.

Hence the larger the sample size $N$, the higher the probability that problem SA provides a feasible solution to problem SLS. However, using a large sample size $N$ means introducing a large number of binary variables $\alpha^{i}$ and of big- $M$ type constraints (28) in the formulation. Thus, even if problem SA is a mixed-integer linear program, its resolution by a mathematical programming solver poses some computational difficulty in practice. 
Several mixed-integer linear programming techniques have been recently proposed to remedy to this difficulty. Valid inequalities exploiting the fact that, for a given period $t$, constraints (28) define a mixing set subject to the additional cardinality constraint (29) are proposed by Luedtke et al [15] and further improved by Küçükyavuz [12]. Luedtke et al [15] also investigate the use of a strong extended formulation. We carried out some preliminary computational experiments on a set of 50 instances with $T=20, f=50, h=1, C=100, D \sim U[10 ; 50], \epsilon=0.05$ and $N \in\{100,500,1000,2000,5000\}$. Consistent with the results presented by Luedtke et al [15], our numerical results indicated that solving problem SA using the extended formulation leads to a computation time significantly smaller than solving it using the valid inequalities proposed by Luedtke et al [15] and Küçükyavuz [12]. We thus used this extended formulation in our computational experiments.

\subsection{Strong extended MILP formulation}

We describe in this subsection how the strong extended MILP formulation proposed by Luedtke et al [15] can be used to ease the resolution of problem SA by a MILP solver.

We first define some additional notations.

- For each period $t$, the scenarios are sorted in the non-increasing order of the cumulative demand $D C_{t}^{i}$. Let $\mu_{t}^{j}$ be the index of the scenario in position $j$ in the ordering corresponding to period $t$. We thus have:

$D C_{t}^{\mu_{t}^{1}} \geq D C_{t}^{\mu_{t}^{2}} \geq \ldots \geq D C_{t}^{\mu_{t}^{j}} \geq \ldots \geq D C_{t}^{\mu_{t}^{N}}$.

- Let $p=\lfloor N \epsilon\rfloor$. $p$ is equal to the maximum number of scenarios in which one or more demand satisfaction constraints may be violated by the production plan in any feasible solution.

We then introduce a new set of binary variables denoted by $\beta_{t}^{i}$. $\beta_{t}^{i}$ is defined by: $\beta_{t}^{i}=1$ if the demand satisfaction constraint in period $t$ is violated in scenario $i, \beta_{t}^{i}=0$ otherwise.

As noted by Luedtke et al [15], in order to obtain a feasible solution to problem SA, the number of scenarios for which the demand satisfaction constraint in period $t$ is violated should stay below $p$, i.e. $\sum_{i=1}^{N} \beta_{t}^{i} \leq p$. Moreover, as $D C_{t}^{\mu_{t}^{j}} \geq D C_{t}^{\mu_{t}^{j+1}}, \forall j=1 \ldots N$, we have: $\beta_{t}^{\mu_{t}^{j}} \geq \beta_{t}^{\mu_{t}^{j+1}}, \forall j=1 \ldots N$. This implies that for $j=p+1 \ldots N, \beta_{t}^{\mu_{t}^{j}}$ must be equal to 0 in any feasible solution of problem SA. We therefore only introduce in the extended formulation the $p T$ variables $\beta_{t}^{\mu_{t}^{j}}, t=1 \ldots T, j=1 \ldots p$.

Using this notation, problem SA can be reformulated using the following extended formulation denoted by SAExt. Note that the optimal value of formulation SAExt, $Z_{S A E x t}^{*}$, is equal to $Z_{S A}^{*}$, the optimal value of formulation SA. 


$$
\begin{cases}Z_{S A E x t}^{*}=\min \sum_{t=1}^{T} f_{t} y_{t}+\sum_{t=1}^{T} h_{t}\left(\sum_{\tau=1}^{t} x_{\tau}-\mathbb{E}\left[D C_{t}\right]\right) & \\ x_{t} \leq c_{t} y_{t} & \forall t \\ \sum_{\tau=1}^{t} x_{\tau}+\sum_{j=1}^{p}\left(D C_{t}^{\mu_{t}^{j}}-D C_{t}^{\mu_{t}^{j+1}}\right) \beta_{t}^{\mu_{t}^{j}} \geq D C_{t}^{\mu_{t}^{1}} & \forall t \\ \beta_{t}^{\mu_{t}^{j}} \geq \beta_{t}^{\mu_{t}^{j+1}} & \forall t, \forall j=1 \ldots p \\ \alpha^{\mu_{t}^{j}} \geq \beta_{t}^{\mu_{t}^{j}} & \forall t, \forall j=1 \ldots p \\ \sum_{i=1}^{N} \alpha^{i} \leq\lfloor N \epsilon\rfloor & \\ x_{t} \geq 0 & \\ y_{t} \in\{0,1\} & \forall t \\ \alpha^{i} \in\{0,1\} & \forall t \\ \beta_{t}^{\mu_{t}^{j}} \in\{0,1\} & \forall i=1 \ldots N\end{cases}
$$

Constraints (35) are the demand satisfaction constraints. They state that the cumulative production over interval $[1 ; t]$ should be greater that the largest possible value for the cumulative demand over this interval, $D C_{t}^{\mu_{t}^{1}}$, unless some scenarios display a violation in period $t$. In this latter case, the term $\sum_{j=1}^{p}\left(D C_{t}^{\mu_{t}^{j}}-\right.$ $\left.D C_{t}^{\mu_{t}^{j+1}}\right) \beta_{t}^{\mu_{t}^{j}}$ computes the corresponding allowed decrease in the cumulative demand to be satisfied. Constraints (36) ensure consistency between the variables $\beta$ corresponding to the same period $t$. Constraints (37) link variables $\alpha$ and variables $\beta$ corresponding to the same scenario: they ensure that a scenario is considered as violated as soon as one of the $T$ corresponding demand satisfaction constraints is violated.

Using formulation SAExt instead of formulation SA to solve the mixed-integer linear program obtained in the sample approximation approach leads to a significantly reduced computation time (see Luedtke et al [15]). This is mainly explained by the fact that the lower bounds provided by the linear relaxation of SAExt are significantly stronger that the ones provided by the linear relaxation of SA. As a result, the number of nodes explored by the Branch \& Bound type procedure before finding a guaranteed optimal solution is significantly reduced.

\subsection{Valid inequalities for the deterministic single-item lot-sizing problem}

Formulation SAExt can be further strengthened thanks to the use of valid inequalities available for deterministic single-item lot-sizing problems.

Namely, constraints (35) imply that $\sum_{\tau=1}^{t} x_{\tau} \geq D C_{t}^{\mu_{t}^{p+1}}, \forall t$. Hence there is a deterministic non-negative lower bound for the cumulative production over the interval $[1 ; t]$, i.e. a minimum deterministic cumulative demand to be satisfied by the production plan. This deterministic demand can be used as a starting point 
to generate valid inequalities as would be done for a deterministic single-item lot-sizing problem.

The determination of the most relevant valid inequalities to be used to strengthen formulation SAExt depends on the features of the problem parameters (more precisely on the costs and production capacity). We refer the reader to Pochet and Wolsey [16] for a full introduction to this field.

In our computational experiments, we assume a constant production capacity, i.e. $c_{t}=c, \forall t$ and use the valid inequalities proposed by Leung et al [13] for the deterministic single-item lot-sizing problem with a constant production capacity. These valid inequalities are added to formulation SAExt at the root of the Branch $\&$ Bound search tree thanks to a cutting-plane generation algorithm based on the polynomial separation algorithm described in [13].

\section{Partial sample approximation approach}

As will be shown by the computational results presented in Section 7, the sample approximation approach, even when using the extended formulation SAExt strengthened by lot-sizing valid inequalities, fails at providing feasible solutions of problem SLS for small samples and leads to a significant computation time when the sample size $N$ increases. This is why we propose in what follows to use a new extension of this approach. This extension is based on the assumption that the demand in the first period $D_{1}$ is statistically independent of the demand in the other periods. Note that $D_{1}$ plays a special role in the joint chance constraint (16) as this is the only random variable appearing in all the demand satisfaction constraints. Similarly to the sample approximation approach, the proposed extension relies on a Monte Carlo sampling method. However, the sampling is not carried out on all the random variables involved in the stochastic problem (i.e. on $D_{1}, D_{2}$, $\ldots, D_{T}$ ) but only on part of them (more precisely on $D_{2}, \ldots, D_{T}$ in our case). We thus refer to it as the partial sample approximation approach.

We first explain in Subsection 6.1 how the proposed method leads to the formulation of a new chance-constrained program featuring a series of $N$ joint chance constraints, each one involving a single random variable $D_{1}$. We then focus on two special cases: the case where $D_{1}$ follows a uniform distribution (see Subsection 6.2) and the case where $D_{1}$ follows a normal distribution (see Subsection 6.3). We show how, in both cases, the use of a conservative convex approximation of the feasible set defined by each of the $N$ joint chance constraints leads to the formulation of a deterministic MILP involving the same number of binary variables as the original problem SLS. We eventually discuss in Subsection 6.4 the definition of non trivial values for the minimum deterministic cumulative demand and their use to ease the resolution of the obtained MILP.

\subsection{General case}

Let $\Delta C=\left(0, D_{2}, \ldots, \sum_{\tau=2}^{t} D_{\tau}, \ldots, \sum_{\tau=2}^{T} D_{\tau}\right)$ be the random vector representing the cumulative demand over periods 2 to $t$, for each period $t=1 \ldots T$. We con-

sider a Monte Carlo sample of vector $\Delta C$ and denote $\Delta C^{1}, \ldots, \Delta C^{i}, \ldots, \Delta C^{N}$ the corresponding $N$ independent and identically distributed sampled vectors. 
The partial sample approximation approach relies on the idea that, given a production plan $x$, the value of the joint probability involved in constraint (16) can be approximately computed as follows:

$$
\begin{aligned}
\operatorname{Pr}\left(\sum_{\tau=1}^{t} x_{\tau} \geq D C_{t}, \forall t\right) & =\operatorname{Pr}\left(\sum_{\tau=1}^{t} x_{\tau}-\Delta C_{t} \geq D_{1}, \forall t\right) \\
& =\mathbb{E}\left[\mathbb{I}\left(\sum_{\tau=1}^{t} x_{\tau}-\Delta C_{t} \geq D_{1}, \forall t\right)\right] \\
& =\mathbb{E}_{\Delta C} \mathbb{E}_{D_{1}}\left[\mathbb{I}\left(\sum_{\tau=1}^{t} x_{\tau}-\Delta C_{t} \geq D_{1}, \forall t\right)\right] \\
& =\mathbb{E}_{\Delta C}\left[\operatorname{Pr}\left(\sum_{\tau=1}^{t} x_{\tau}-\Delta C_{t} \geq D_{1}, \forall t\right)\right] \\
& \approx \frac{1}{N} \sum_{i=1}^{N} \operatorname{Pr}\left(\sum_{\tau=1}^{t} x_{\tau}-\Delta C_{t}^{i} \geq D_{1}, \forall t\right)
\end{aligned}
$$

Equalities (44) and (46) rely on the fact that the probability of an event is equal to the expected value of an indicator function that is one if the event has occurred and zero otherwise. Equality (45) makes use of the assumption that $D_{1}$ is statistically independent of $\Delta C$ to decompose the computation of the expected value in two parts. The expected value appearing in (46) is then approximately computed via a sample average approximation in (47). The idea underlying the proposed partial sample approximation approach can thus be understood as follows. For each scenario $i$, the probability $\pi^{i}=\operatorname{Pr}\left(\sum_{\tau=1}^{t} x_{\tau}-\Delta C_{t}^{i} \geq D_{1}, \forall t\right)$ that no stockout occurs during the whole planning horizon in case the realized demand over periods 2 to $T$ corresponds to the sampled vector $\Delta C^{i}$ is computed. The expected value of $\pi^{i}$ over all scenarios, i.e. $\sum_{i=1}^{N} \pi^{i} / N$, is then used as an estimation of the value of the joint probability. We refer the reader to Cheng et al [7] for a more thorough mathematical discussion on this approximation of the joint probability.

This approximation enables us to reformulate problem SLS as a new joint chance-constrained program. This is done by introducing the decision variables $\pi^{i}, i=1 \ldots N . \pi^{i} \in[0 ; 1]$ represents the probability that no stock-out occurs during the whole planning horizon in case the realized demand over periods 2 to $T$ is equal to the sampled vector $\Delta C^{i}$. Note that, contrary to what is done in the sample approximation where a binary variable $\alpha^{i}$ has to be introduced for each scenario $i$, the variables $\pi^{i}$ introduced in the partial sample approximation approach are continuous variables.

This leads to the following chance-constrained program denoted by PSA in the sequel of the paper. 


$$
\begin{cases}Z_{P S A}^{*}=\min \sum_{t=1}^{T} f_{t} y_{t}+\sum_{t=1}^{T} h_{t}\left(\sum_{\tau=1}^{t} x_{\tau}-\mathbb{E}\left[D C_{t}\right]\right) & \\ x_{t} \leq c_{t} y_{t} & \forall t \\ \pi^{i}=\operatorname{Pr}\left(\sum_{\tau=1}^{t} x_{\tau}-\Delta C_{t}^{i} \geq D_{1}, \forall t=1 \ldots T\right) & \forall i=1 \ldots N \\ \frac{1}{N} \sum_{i=1}^{N} \pi^{i} \geq 1-\epsilon & \\ x_{t} \geq 0 & \forall t \\ y_{t} \in\{0,1\} & \forall t \\ \pi^{i} \in[0 ; 1] & \forall i=1 \ldots N\end{cases}
$$

Constraints (50) compute the probability that all demand satisfaction constraints are satisfied by the production plan $x$ in the scenario $i$. (51) is the joint probability constraint ensuring that the expected value of $\pi^{i}$ over all scenarios is above the minimum acceptable value.

We note that, similarly to the sample approximation approach, problem PSA is based on an approximate representation of constraint (16) of problem SLS. There is thus no definite guarantee that it will provide feasible solutions of problem SLS. However, Cheng et al [7] show that the partial sample approximation approach has the same convergence properties as the sample approximation approach. For instance, under certain conditions, the optimal value of problem PSA converges to the optimal value of problem SLS with probability one when $N$ tends to infinity.

Moreover, we would like to point out that formulation PSA involves a series of $N$ joint chance-constraints (50). However, contrary to the initial constraint (16), these constraints involve a single random variable $D_{1}$ and thus may be easier to handle, depending on the probability distribution of $D_{1}$. We refer the reader to Cheng et al [7] for a general discussion on how to handle the probabilistic constraints (50) for a variety of probability distributions and focus in what follows on two special cases.

\subsection{Special case: $D_{1} \sim U\left[L_{1} ; U_{1}\right]$}

We assume here that the random variable $D_{1}$ follows a uniform distribution on an interval denoted by $\left[L_{1} ; U_{1}\right]$. In this case, problem PSA can be reformulated as an equivalent deterministic mixed-integer linear program (see Subsection 6.2.1) but this requires the introduction of $N$ additional binary variables. We thus consider solving a conservative approximation of problem PSA in which no additional binary variables are needed (see Subsection 6.2.2). This approximation relies on the use of a convex conservative approximate representation of the feasible set defined by each joint chance-constraint (50).

\subsubsection{Equivalent deterministic mixed-integer linear program}

When $D_{1} \sim U\left[L_{1} ; U_{1}\right]$, the value of the probability $\pi^{i}$ can be computed as follows: 


$$
\pi^{i}= \begin{cases}0 & \text { if } \min _{t}\left(\sum_{\tau=1}^{t} x_{\tau}-\Delta C_{t}^{i}\right)<L_{1} \\ \frac{\min _{t}\left(\sum_{\tau=1}^{t} x_{\tau}-\Delta C_{t}^{i}\right)-L_{1}}{U_{1}-L_{1}} & \text { if } \min _{t}\left(\sum_{\tau=1}^{t} x_{\tau}-\Delta C_{t}^{i}\right) \in\left[L_{1} ; U_{1}\right] \\ 1 & \text { if } \min _{t}\left(\sum_{\tau=1}^{t} x_{\tau}-\Delta C_{t}^{i}\right)>U_{1}\end{cases}
$$

We introduce a new set of binary variables $\gamma^{i}$ defined by: $\gamma^{i}=1$ if $\min _{t}\left(\sum_{\tau=1}^{t} x_{\tau}-\right.$ $\left.\Delta C_{t}^{i}\right)<L_{1}$ and $\gamma^{i}=0$ otherwise. This leads to the formulation of a deterministic mixed-integer linear program equivalent to problem PSA. This formulation is denoted by PSA_U in the sequel of the paper.

$$
\begin{cases}Z_{P S A_{-} U}^{*}=\min \sum_{t=1}^{T} f_{t} y_{t}+\sum_{t=1}^{T} h_{t}\left(\sum_{\tau=1}^{t} x_{\tau}-\mathbb{E}\left[D C_{t}\right]\right) & \\ x_{t} \leq c_{t} y_{t} & \forall t \\ \pi^{i} \leq \frac{\sum_{\tau=1}^{t} x_{\tau}-\Delta C_{t}^{i}-L_{1}}{U_{1}-L_{1}}+\frac{\Delta C_{t}^{i}+L_{1}}{U_{1}-L_{1}} \gamma^{i} & \forall i=1 \ldots N, \forall t \\ \pi^{i} \geq 0 & \forall i=1 \ldots N \\ \pi^{i} \leq 1-\gamma^{i} & \forall i=1 \ldots N \\ \sum_{\tau=1}^{t} x_{\tau} \geq\left(\Delta C_{t}^{i}+L_{1}\right)\left(1-\gamma^{i}\right) & \forall i=1 \ldots N, \forall t \\ \frac{1}{N} \sum_{i=1}^{N} \pi^{i} \geq 1-\epsilon & \\ x_{t} \geq 0 & \forall t \\ y_{t} \in\{0,1\} & \forall t \\ \gamma^{i} \in\{0,1\} & \forall i=1 \ldots N\end{cases}
$$

Constraints (57)-(59) compute the value of the probability $\pi^{i}$ for each scenario $i$. In case $\gamma^{i}=0$, constraints (57) and (59) make sure that $\pi^{i} \leq$ $\min \left(\min _{t}\left(\frac{\sum_{\tau=1}^{t} x_{\tau}-\Delta C_{t}^{i}-L_{1}}{U_{1}-L_{1}}\right) ; 1\right)$. In case $\gamma^{i}=1$, constraints (57) become inactive and constraints (58)-(59) guarantee that $\pi^{i}=0$. Constraints (60) ensure that $\gamma^{i}=1$ if $\min _{t}\left(\sum_{\tau=1}^{t} x_{\tau}-\Delta C_{t}^{i}\right)<L_{1}$ and $\gamma^{i}=0$ otherwise.

Similarly to formulation SA, formulation PSA_U can be strengthened by using disaggregated decision variables defined as: $\delta_{t}^{i}=1$ if $\sum_{\tau=1}^{t} x_{\tau}-\Delta C_{t}^{i}-L_{1}<0$, $\delta_{t}^{i}=0$ otherwise. Let $\left(\nu_{t}^{1}, \ldots, \nu_{t}^{j}, \ldots, \nu_{t}^{N}\right)$ be an ordering of the scenarios in the nondecreasing order of the cumulative demand $\Delta C_{t}^{i}$. Using the same considerations as in Subsection 5.2, we can set $\delta_{t}^{\nu_{t}^{j}}$ to 0 for all $j=p+1 \ldots N$.

This leads to the following extended formulation denoted by PSAExt_U . Note that its optimal value, $Z_{P S A E x t_{-} U}^{*}$, is equal to the optimal value of formulation PSA_U, $Z_{P S A_{-} U}^{*}$. 


$$
\begin{cases}Z_{P S A E x t_{-} U}^{*}=\min \sum_{t=1}^{T} f_{t} y_{t}+\sum_{t=1}^{T} h_{t}\left(\sum_{\tau=1}^{t} x_{\tau}-\mathbb{E}\left[D C_{t}\right]\right) & \\ x_{t} \leq c_{t} y_{t} & \forall t \\ \pi^{i} \leq \frac{\sum_{\tau=1}^{t} x_{\tau}-\Delta C_{t}^{i}-L_{1}}{U_{1}-L_{1}}+\frac{\Delta C_{t}^{i}+L_{1}}{U_{1}-L_{1}} \gamma^{i} & \forall i=1 \ldots N, \forall t(67) \\ \pi^{i} \geq 0 & \forall i=1 \ldots N \\ \pi^{i} \leq 1-\gamma^{i} & \forall i=1 \ldots N \\ \sum_{\tau=1}^{t} x_{\tau}+\sum_{j=1}^{p}\left(\Delta C_{t}^{\nu_{t}^{j}}-\Delta C_{t}^{\nu_{t}^{j+1}}\right) \delta_{t}^{\nu_{t}^{j}} \geq \Delta C_{t}^{\nu_{t}^{1}}+L_{1} & (68) \\ \delta_{t}^{\nu_{t}^{j+1}} \leq \delta_{t}^{\nu_{t}^{j}} & \forall t \\ \delta_{t}^{\nu_{t}^{j}} \leq \gamma^{\nu_{t}^{j}} & \forall j=1 \ldots p, \forall t(71) \\ \frac{1}{N} \sum_{i=1}^{N} \pi^{i} \geq 1-\epsilon & \forall j=1 \ldots p, \forall t(72) \\ x_{t} \geq 0 & \\ y_{t} \in\{0,1\} & \forall t \\ \gamma^{i} \in\{0,1\} & \forall t \\ \delta_{t}^{\nu_{t}^{j}} \in\{0,1\} & \forall i=1 \ldots N\end{cases}
$$

As will be shown by the computational results presented in Section 7, formulation PSAExt_U requires prohibitively long computation times for large sample sizes. We thus investigate in the next subsection a conservative approximation of problem PSA, in which no additional binary variable is introduced.

\subsubsection{Conservative approximate deterministic mixed-integer linear program}

The proposed conservative approximation of problem PSA relies on the following approximate computation of probability $\pi^{i}$ :

$$
\pi^{i} \approx \tilde{\pi}^{i}= \begin{cases}\frac{\min _{t}\left(\sum_{\tau=1}^{t} x_{\tau}-\Delta C_{t}^{i}\right)-L_{1}}{U_{1}-L_{1}} & \text { if } \min _{t}\left(\sum_{\tau=1}^{t} x_{\tau}-\Delta C_{t}^{i}\right) \leq U_{1} \\ 1 & \text { if } \min _{t}\left(\sum_{\tau=1}^{t} x_{\tau}-\Delta C_{t}^{i}\right)>U_{1}\end{cases}
$$

Note that in this approximation, the value of the probability is computed exactly (i.e. $\left.\pi^{i}=\tilde{\pi}^{i}\right)$ in case $\min _{t}\left(\sum_{\tau=1}^{t} x_{\tau}-\Delta C_{t}^{i}\right) \geq L_{1}$ and underestimated (i.e. $\left.\tilde{\pi}^{i} \leq \pi^{i}\right)$ in case $\min _{t}\left(\sum_{\tau=1}^{t} x_{\tau}-\Delta C_{t}^{i}\right)<L_{1}$. Namely, in this latter case, $\tilde{\pi}^{i}$ has a negative value whereas $\pi^{i}$ is equal to 0 .

Thanks to the use of this approximate computation of probability $\pi^{i}$, it is possible to reformulate problem PSA as a mixed-integer linear program without introducing the binary variables $\gamma^{i}$. This problem is denoted by PSACons_U in the sequel of the paper. 


$$
\begin{cases}Z_{P S A C o n s_{-} U}^{*}=\min \sum_{t=1}^{T} f_{t} y_{t}+\sum_{t=1}^{T} h_{t}\left(\sum_{\tau=1}^{t} x_{\tau}-\mathbb{E}\left[D C_{t}\right]\right) & \\ x_{t} \leq c_{t} y_{t} & \forall t \\ \tilde{\pi}^{i} \leq \frac{\sum_{\tau=1}^{t} x_{\tau}-\Delta C_{t}^{i}-L_{1}}{U_{1}-L_{1}} & \forall i, \forall t \\ \tilde{\pi}^{i} \leq 1 & \forall i \\ \frac{1}{N} \sum_{i=1}^{N} \tilde{\pi}^{i} \geq 1-\epsilon & \\ x_{t} \geq 0 & \forall t \\ y_{t} \in\{0,1\} & \forall t\end{cases}
$$

Note that problem PSACons_U is a conservative (safe) approximation of problem PSA_U. Namely, any feasible solution of problem PSACons_U $(x, y, \tilde{\pi})$ provides a feasible solution $(x, y, \pi)$ of problem PSA_U by putting $\pi^{i}=0$ for each scenario $i$ such that $\tilde{\pi}^{i}<0$. As we have $\pi^{i} \geq \tilde{\pi}^{i}, \forall i=1 \ldots N$, we have $\frac{1}{N} \sum_{i=1}^{N} \pi^{i} \geq \frac{1}{N} \sum_{i=1}^{N} \tilde{\pi}^{i}$. Hence, a production plan $(x, y)$ which satisfies constraint (82) also satisfies constraint (61) and $(x, y, \pi)$ is a feasible solution of problem PSA_U.

\subsection{Special case : $D_{1} \sim \mathcal{N}\left(m_{1}, s_{1}\right)$}

We now consider the case where $D_{1}$ follows a normal distribution with mean $m_{1}$ and standard deviation $s_{1}$. The normal distribution is widely used to represent the demand and the forecasting errors in standard inventory management and forecasting models although it has the drawback of placing some probability on unrealistic negative demand values. The standard models usually assume that this probability is sufficiently small to be negligible. Namely, the probability of getting a negative value with a normal distribution is at most $2.5 \%$ when the ratio $m_{1} / s_{1}$ is equal to 2 and falls below $0.15 \%$ when $m_{1} / s_{1}$ is greater than 3 . In practice, such large values of $m_{1} / s_{1}$ can be found for high volume products displaying a demand with a large expected value and a low variability.

In this subsection, we discuss a conservative convex approximation of problem PSA in the case where $D_{1}$ follows a normal distribution. This approximation makes use of the fact that the cumulative probability function $F_{D_{1}}$ is convex over ] $\left.\infty ; m_{1}\right]$ and concave over the interval $\left[m_{1},+\infty[\right.$.

This conservative piecewise linear approximation of $F_{D_{1}}$, denoted by $\tilde{F}_{D_{1}}(X)$, uses a set of $B+1$ breakpoints $\phi_{b}, b=0 \ldots B$ such that $\phi_{0}=m_{1}, \phi_{b}>\phi_{b-1}, \forall b=$ 1 ...B. We define:

$$
F_{D_{1}}(X) \approx \tilde{F}_{D_{1}}(X)= \begin{cases}\sigma_{0} X+\theta_{0} & \text { if } X \leq \phi_{0} \\ \sigma_{b} X+\theta_{b} & \text { if } X \in\left[\phi_{b-1} ; \phi_{b}\right], \forall b=1 \ldots B \\ \sigma_{B} \phi_{B}+\theta_{B} & \text { if } X \geq \phi_{B}\end{cases}
$$

where the slope and intercept of each segment are computed as follows: 


$$
\left\{\begin{array}{l}
\sigma_{0}=F_{D_{1}}^{\prime}\left(\phi_{0}\right) \text { and } \theta_{0}=F_{D_{1}}\left(\phi_{0}\right)-\sigma_{0} \phi_{0} \\
\sigma_{b}=\frac{F_{D_{1}}\left(\phi_{b}\right)-F_{D_{1}}\left(\phi_{b-1}\right)}{\phi_{b}-\phi_{b-1}} \text { and } \theta_{b}=F_{D_{1}}\left(\phi_{b}\right)-\sigma_{b} \phi_{b} \quad \forall b=1 \ldots B
\end{array}\right.
$$

Note that we have $F_{D_{1}}(X) \geq \tilde{F}_{D_{1}}(X), \forall X$. Namely:

- $\left[X \mapsto \sigma_{0} X+\theta_{0}\right]$ is the tangent line to the curve $\left[X \mapsto F_{D_{1}}(X)\right]$ at point $m_{1}$. As $F_{D_{1}}$ is convex over ] $\left.-\infty ; m_{1}\right]$, we have: $F_{D_{1}}(X) \geq \sigma_{0} X+\theta_{0}, \forall X \leq \phi_{0}$.

- $\left[X \mapsto \sigma_{b} X+\theta_{b}\right], b=1 \ldots B$ are chords between two points $\phi_{b-1}$ and $\phi_{b}$ belonging $\left[m_{1},+\infty\left[\right.\right.$. As $F_{D_{1}}$ is concave over this interval, we have: $F_{D_{1}}(X) \geq \sigma_{b} X+$ $\theta_{b}, \forall X \geq \phi_{0}, \forall b=1 \ldots B$.

$-F_{D_{1}}$ is strictly increasing over $\mathbb{R}$. This implies $F_{D_{1}}(X) \geq F_{D_{1}}\left(\phi_{B}\right)=\sigma_{B} \phi_{B}+$ $\theta_{B}, \forall X \geq \phi_{B}$.

This allows us to reformulate problem PSA as the following mixed-integer linear program denoted by PSACons_N.

$$
\begin{cases}Z_{P S A C o n s_{-} N}^{*}=\min \sum_{t=1}^{T} f_{t} y_{t} & \\ \qquad \sum_{t=1}^{T} h_{t}\left(\sum_{\tau=1}^{t} x_{\tau}-\mathbb{E}\left[D C_{t}\right]\right) & \\ x_{t} \leq c_{t} y_{t} & \forall t \\ \tilde{\pi}^{i} \leq \sigma_{b}\left(\sum_{\tau=1}^{t} x_{\tau}-\Delta C_{t}^{i}\right)+\theta_{b} & \forall i, \forall t, \forall b=0 \ldots B \\ \tilde{\pi}^{i} \leq \sigma_{B} \phi_{B}+\theta_{B} & \forall i \\ \frac{1}{N} \sum_{i=1}^{N} \tilde{\pi}^{i} \geq 1-\epsilon & \\ x_{t} \geq 0 & \forall t \\ y_{t} \in\{0,1\} & \forall t\end{cases}
$$

Note how constraints (92)-(93) compute the value of the probabilities in the approximation, $\tilde{\pi}^{i}$, via a set of linear inequalities.

\subsection{Formulation improvements}

We now investigate the determination of a non trivial value for the minimum deterministic cumulative demand to be satisfied by any feasible solution of problem SLS. This information will be used to ease the resolution of problems PSAExt_U, PSACons_U and PSACons_N by a MILP solver through: (i) the reduction of the MILP size thanks to the early detection of redundant constraints, (ii) the formulation strengthening through valid inequalities available for deterministic single-item capacitated lot-sizing. 
Proposition 1 Consider a production plan $(x, y)$ feasible for problem SLS. Let $F_{D_{1}}$ be the cumulative probability distribution of the random variable $D_{1}$.

- for $t=1$, we have:

$$
x_{1} \geq F_{D_{1}}^{-1}(1-\epsilon)
$$

- for a given period $t \in[2 ; T]$

Let $\left(\nu_{t}^{1}, \ldots, \nu_{t}^{j}, \ldots, \nu_{t}^{N}\right)$ be an ordering of the scenarios in the non-decreasing order of the cumulative demand $\Delta C_{t}^{i}$.

Let $k_{t}$ be the index of the first scenario in this ordering such that:

$$
\sum_{i=1}^{N} F_{D_{1}}\left(F_{D_{1}}^{-1}(1-\epsilon)+\Delta C_{t}^{\nu_{t}^{k_{t}}}-\Delta C_{t}^{i}\right)<N(1-\epsilon)
$$

We have:

$$
\sum_{\tau=1}^{t} x_{\tau} \geq F_{D_{1}}^{-1}(1-\epsilon)+\Delta C_{t}^{\nu_{t}^{k_{t}}}
$$

Proof See appendix.

Proposition 1 defines a minimum deterministic cumulative demand $d c_{t}^{\text {min }}=$ $F_{D_{1}}^{-1}(1-\epsilon)+\Delta C_{t}^{\nu_{t}^{k_{t}}}$ which has to be satisfied by any feasible production plan.

This can first be used to significantly reduce the size of the MILP to be solved as some constraints or variables become redundant. More precisely,

- In formulation PSAExt_U, we can set to 0 all binary variables $\delta_{t}^{i}$ such that $d c_{t}^{m i n}-\Delta C_{t}^{i}-L_{1} \geq 0$ and all binary variables $\gamma^{i}$ such that $\delta_{t}^{i}=0, \forall t$.

- In formulation PSACons_U, in each period $t$, constraints (80) and (81) are redundant for all scenarios $i$ such that $\frac{d c_{t}^{m i n}-\Delta C_{t}^{i}-L_{1}}{U_{1}-L_{1}} \geq 1$.

- In formulation PSACons_N, for each period $t$ and each segment $b$, constraints (92) are redundant for all scenarios $i$ such that $d c_{t}^{\text {min }}-\Delta C_{t}^{i} \geq \phi_{b}$.

Second, similarly to what is done to strengthen formulation SAExt for the sample approximation approach, the minimum deterministic cumulative demand $d c_{t}^{\min }$ can be used as a starting point to generate valid inequalities such as the ones proposed by Leung et al [13] for the deterministic single-item lot-sizing problem with a constant production capacity.

\section{Numerical results}

We discuss in this Section the results of some computational experiments carried out on small to medium size instances of the problem. The main objective of these experiments is to assess the effectiveness of the proposed partial sample approximation approach by comparing it with the Bonferroni conservative approximation and the sample approximation approach. 


\subsection{Instances}

We generated test problems by considering a planning horizon of $T \in\{10,20,30\}$ periods. In each problem, the inventory holding and setup costs are assumed to be time-independent and are set to $h=1$ and $f \in\{25,50,75\}$. Similarly, the production capacity is assumed to be time-independent and is set to $c \in\{50,100,150\}$.

Four different cases are considered for the random demands $D_{t}, t=1 \ldots T$.

$-D_{t}, t=1 \ldots T$ are statistically independent of one another and follow:

- a uniform distribution on the interval $[L, U] \in\{[20 ; 40],[10 ; 50],[0 ; 60]\}$.

- a normal distribution of parameter $m=30$ and $s \in\{5,10,15\}$.

$-D_{t}, t=1 \ldots T$ are statistically dependent and are generated according to an autoregressive process of type AR1: $D_{t}=\rho D_{t-1}+\chi(1-\rho)+r(t)$ where $\rho \in\{-1,-0.5,0,0.5,1\}$ is the first order autocorrelation coefficient, $\chi=30$ and the residual $r(t)$ follows:

- a uniform distribution on the interval $[L, U]=[-20,20]$.

- a normal distribution with mean $m=0$ and standard deviation $s=10$.

The size of the sample obtained by the Monte Carlo method, $N$, is varied between 100 and 5000 scenarios: $N \in\{100,500,1000,2000,5000\}$. Note that, when the demand is generated from a normal distribution, it may happen in a few cases that the sampled value $D_{t}^{i}$ takes a negative value. When this happens, we set the value of $D_{t}^{i}$ to 0 to avoid introducing unrealistic negative demands in the sampled scenarios.

Five possible values are considered for the maximum acceptable risk level: $\epsilon \in\{0.15,0.10,0.05,0.02,0.01\}$.

We defined two reference sets of 10 instances in which $T=20, h=1, f=50$, $c=100, N=1000, \epsilon=0.05, D_{t}, t=1 \ldots T$ are statistically independent and follow either a uniform distribution on the interval $[10,50]$ or a normal distribution with mean $m=30$ and $s=10$. We then created additional sets of instances, each time changing the value of a single parameter in order to assess its impact on the problem resolution. For each considered set, 10 samples of scenarios, i.e. 10 instances, were randomly generated, leading to a total of 420 instances.

All tests were run on an Intel Core i5 $(2.6 \mathrm{GHz})$ with 4 Go of RAM, running under Windows 7. We used the mathematical programming solver CPLEX 12.6 with the default settings to solve the problem using the MILP formulations discussed in Sections 4 to 6 . The piecewise linear approximation of $F_{D_{1}}$ used in formulation PSACons_N was built with $B=4, \phi_{0}=m_{1}, \phi_{1}=m_{1}+0.5 s_{1}, \phi_{2}=m_{1}+s_{1}$, $\phi_{3}=m_{1}+1.5 s_{1}$ and $\phi_{4}=m_{1}+3 s_{1}$.

\subsection{Numerical results: independent random variables $D_{t}, t=1 \ldots T$}

We first focus on the case where the random variables $D_{t}, t=1 \ldots T$ are statistically independent of one another and follow either a uniform distribution or a normal distribution. The instances in which $D_{t}, t=1 \ldots T$ follow a uniform distribution are solved using formulations BON, SAExt, PSAExt_U and PSACons_U. The instances in which $D_{t}, t=1 \ldots T$ follow a normal distribution are solved using formulations BON, SAExt and PSACons_N. 
Tables 1 to 6 display the corresponding results. Each line corresponds to the average value over the corresponding 10 instances. We provide for each set of 10 instances:

- Bin: the average number of binary variables in the formulation.

- Cons: the average number of constraints in the formulation.

- Cost: the average value of the optimal solution of the corresponding MILP.

- Prob: the average value of the probability $\operatorname{Pr}\left(\sum_{\tau=1}^{t} x_{\tau}^{*} \geq D C_{t}, \forall t=1 \ldots T\right)$ where $x^{*}$ is the optimal solution of the MILP formulation. Prob corresponds to a post-optimization estimation of the joint probability. It is is obtained by using a sample of $N_{c}=100000$ scenarios different from the ones used in the optimization phase. For each instance, we count the number $N_{s}$ of scenarios for which all demand satisfaction constraints are satisfied by the corresponding production plan $x^{*}$. Prob is then computed as the proportion $N_{s} / N_{c}$ of scenarios for which there is no violation. We consider that $x^{*}$ is a feasible solution of problem SLS in case Prob is greater or equal to $1-\epsilon$ and an infeasible solution otherwise.

- Feas: the number of instances (out of the 10 corresponding ones) for which the production plan $x^{*}$ is a feasible solution of problem SLS (i.e. is such that Prob $\geq 1-\epsilon)$. Note that Feas is not defined for formulation BON as this formulation does not use the sampled scenarios.

- Time: the average computation time needed to solve to optimality the MILP formulation.

Results from Tables 1 to 6 first show that, whether $D_{1}$ follows a uniform or a normal distribution, formulation BON based on the Bonferroni approximation is capable of providing feasible solutions of problem SLS within very short computation times (less than 1s in most cases). However, these solutions are rather conservative. The value of Prob is namely significantly higher than the required value $1-\epsilon$ in all cases. As a result, these solutions are significantly more expensive than the solutions provided by the other formulations: for instance, the solutions of formulation BON are on average $24 \%$ more expensive than the ones of formulation SAExt.

Second, for the case where $D_{1}$ follows a uniform distribution, problem PSA can be reformulated into an equivalent deterministic mixed-integer linear program. This enables us to carry out a comparison between formulation SAExt, which is based on the sample approximation approach, formulation PSAExt_U, which solves problem PSA exactly, and formulation PSACons_U, which is a conservative approximation. The numerical results displayed in Tables 1 to 6 show that, out of the 140 studied instances for which PSAExt_U could be solved to optimality within the time limit of 3600 s, a feasible solution was found for 1 instance with formulation SAExt, 44 instances with formulation PSAExt_U and 81 instances with formulation PSACons_U. We thus note that the partial sample approximation method gives more reliable solutions than the sample approximation method, even without using a conservative approximation of $F_{D_{1}}$. This might be explained by the fact that the partial approximation uses an exact representation of the cumulative probability distribution of $D_{1}, F_{D_{1}}$, whereas the sample approximation relies on a discrete approximation obtained by sampling. Moreover, we observe that formulation PSACons_U is significantly more conservative than formulation PSAExt_U as it finds feasible solutions of the stochastic problem for a 
number of instances nearly twice as large as formulation PSAExt_U. However, the average cost increase when using formulation PSACons_U instead of formulation PSAExt_U stays below 2\%, which seems an acceptable threshold. In terms of computation time, formulation PSAExt_U requires prohibitive long computation times and might not be usable in practice. But, the average computation time when using formulation PSACons_U is only $10.5 \mathrm{~s}$ whereas it is $83.3 \mathrm{~s}$ when using formulation SAExt. This might be explained by the fact that formulation PSACons_U involves a number of binary variables significantly smaller than formulation SAExt.

Third, for the case where $D_{1}$ follows a normal distribution, there is no equivalent mixed-integer linear programming formulation of problem PSA. We thus focus on the comparison between formulations SAExt and formulation PSACons_N which is based on a conservative approximation of problem PSA. We first note that formulation SAExt fails at providing feasible solutions of problem SLS for the sample sizes considered in this work. This can be seen by the facts that only 1 of the 170 instances considered in Tables 1 to 6 has a solution satisfying the joint probability constraint (16) when solved with SAExt and that the average value of Prob is below the targeted value $1-\epsilon$ in all cases. In contrast, using formulation PSACons_N, feasible solutions of problem SLS are obtained for 139 out of the 170 instances and Prob is above the targeted value $1-\epsilon$ in most cases. Moreover, the average computation time is decreased from $128.5 \mathrm{~s}$ when using formulation SAExt to 43.6s when using formulation PSACons_N. However, we note that the solutions provided by PSACons_N might be too conservative. Namely, in some cases, the value of Prob is significantly larger than $1-\epsilon$. This might indicate the existence of less expensive feasible solutions of problem SLS which none of the studied approaches is capable of finding.

Finally, results from Tables 1 to 6 also enable us to assess the impact of the various parameters on the problem resolution. They namely show that:

- The computation time of the sample approximation and the partial sample approximation approaches significantly increases with the sample size $N$. However, a minimum sample size is required to ensure a good approximation of the joint probability and to obtain feasible solutions of problem SLS. For the partial sample approximation approach, the value $N=1000$ seems to be an acceptable trade-off. But for the sample approximation approach, a sample size of at least 5000 scenarios seems to be necessary.

- When the value of the maximum acceptable risk level $\epsilon$ decreases, the size of the mixed-integer linear programs obtained with the sample approximation and the partial sample approximation approaches decreases, leading to an overall decrease in the computation time. However, it appears that the smaller $\epsilon$, the more difficult it is to find feasible solutions of problem SLS.

- The horizon length $T$ also has a direct impact of the size of the mixed-integer linear programs so that increasing $T$ leads to longer computation times.

- The values of the ratio $\frac{f}{h}$, of the production capacity $c$ and of the standard deviation of $D_{1}$ appear to have a limited impact on both the number of feasible solutions obtained and the computation time. 
Table 1 Impact of the sample size $N$

\begin{tabular}{|c|c|c|c|c|c|c|c|c|}
\hline$\overline{D_{1}}$ & $\mathrm{~N}$ & Formulation & Bin & Cons & Cost & Prob & Feas & Time \\
\hline \multirow[t]{15}{*}{ Uniform } & \multirow[t]{3}{*}{100} & SAExt & 220 & 241 & 2100.6 & 0.842 & 0 & $1.3 \mathrm{~s}$ \\
\hline & & PSAExt_U & 38 & 477 & 2297.7 & 0.921 & 1 & $3.5 \mathrm{~s}$ \\
\hline & & PSACons_U & 20 & 418 & 2315.1 & 0.924 & 1 & $1.0 \mathrm{~s}$ \\
\hline & \multirow[t]{3}{*}{500} & SAExt & 1020 & 1041 & 2317.8 & 0.926 & 0 & $5.0 \mathrm{~s}$ \\
\hline & & PSAExt_U & 136 & 2093 & 2395.9 & 0.947 & 3 & $58.5 \mathrm{~s}$ \\
\hline & & PSACons_U & 20 & 1830 & 2403.3 & 0.949 & 5 & $2.4 \mathrm{~s}$ \\
\hline & \multirow[t]{3}{*}{1000} & SAExt & 2020 & 2041 & 2343.7 & 0.934 & $\overline{0}$ & $17.1 \mathrm{~s}$ \\
\hline & & PSAExt_U & 241 & 4149 & 2395.9 & 0.949 & 5 & $2423.1 \mathrm{~s}$ \\
\hline & & PSACons_U & 20 & 3651 & 2411.0 & 0.951 & 5 & $7.7 \mathrm{~s}$ \\
\hline & \multirow[t]{3}{*}{2000} & SAExt & 4020 & 4041 & 2342.6 & 0.938 & $\overline{0}$ & $72.0 \mathrm{~s}$ \\
\hline & & PSAExt_U & 473 & 8208 & - & - & - & - \\
\hline & & PSACons_U & 20 & 7188 & 2407.4 & 0.952 & 7 & $19.8 \mathrm{~s}$ \\
\hline & \multirow[t]{3}{*}{5000} & SAExt & 10020 & 10041 & 2363.4 & 0.943 & 0 & $634.1 \mathrm{~s}$ \\
\hline & & PSAExt_U & 1158 & 20501 & - & - & - & - \\
\hline & & PSACons_U & 20 & 17898 & 2407.4 & 0.952 & 9 & $54.2 \mathrm{~s}$ \\
\hline \multirow[t]{10}{*}{ Normal } & \multirow[t]{2}{*}{100} & SAExt & 220 & 241 & 1933.0 & 0.856 & $\overline{0}$ & $1.3 \mathrm{~s}$ \\
\hline & & PSACons_N & 20 & 1108 & 2162.1 & 0.932 & 1 & $1.8 \mathrm{~s}$ \\
\hline & \multirow[t]{2}{*}{500} & SAExt & 1020 & 1041 & 2100.9 & 0.919 & $\overline{0}$ & $6.3 \mathrm{~s}$ \\
\hline & & PSACons_N & 20 & 5102 & 2252.9 & 0.955 & 8 & $8.9 \mathrm{~s}$ \\
\hline & \multirow[t]{2}{*}{1000} & SAExt & 2020 & 2041 & 2140.8 & 0.934 & 0 & $20.3 \mathrm{~s}$ \\
\hline & & PSACons_N & 20 & 10362 & 2265.8 & 0.958 & 10 & $25.1 \mathrm{~s}$ \\
\hline & \multirow[t]{2}{*}{2000} & SAExt & 4020 & 4041 & 2150.6 & 0.937 & 0 & $114.8 \mathrm{~s}$ \\
\hline & & PSACons_N & 20 & 20706 & 2254.2 & 0.957 & 9 & $28.1 \mathrm{~s}$ \\
\hline & \multirow[t]{2}{*}{5000} & SAExt & 10020 & 10041 & 2181.1 & 0.945 & 1 & $695.1 \mathrm{~s}$ \\
\hline & & PSACons_N & 20 & 51443 & 2265.2 & 0.960 & 10 & 309.3s \\
\hline
\end{tabular}

7.3 Numerical results: dependent random variables $D_{t}, t=1 \ldots T$

We now consider the case where there are statistical dependencies between the demand from different periods. Note that the partial sample approximation can take into account any correlations between $D_{2}, \ldots, D_{t}$ as they will be reflected in the generation of the scenarios representing possible realizations of $\Delta C_{2}, \ldots \Delta C_{T}$. However, this approach requires the assumption that $D_{1}$ is statistically independent of $D_{2}, \ldots, D_{t}$. In this subsection, we seek to evaluate whether the partial sample approximation can still be useful in the presence of correlations between $D_{1}$ and $D_{2}, \ldots, D_{t}$.

To achieve this, we use a set of instances in which $D_{t}$ is assumed to follow an autoregressive model of type AR1, i.e. to depend linearly on its previous value $D_{t-1}$ and on a stochastic term. More precisely, we first randomly generated the value of $D_{1}$, assuming either a uniform distribution on $[10,50]$ or a normal distribution distribution with mean 30 and standard deviation 10 . We then generated the value of $D_{t}$, for periods $2 \ldots \mathrm{T}$, following the model $D_{t}=\rho D_{t-1}+\chi(1-\rho)+r(t)$ where $\rho \in\{-1,-0.5,0,0.5,1\}, \chi=30$ and $r(t)$ follows either a uniform distribution on the interval $[L, U]=[-20,20]$ or a normal distribution with mean 0 and standard deviation 10. $\rho$ is the first order autocorrelation coefficient. $\rho=0$ means that there is no correlation between $D_{t-1}$ and $D_{t}, \rho=1$ (resp. $\rho=-1$ ) indicates that there is a total positive (resp. negative) correlation between $D_{t-1}$ and $D_{t}$.

The corresponding numerical results are displayed in Table 7 . They first show that the presence of positive or negative correlations between variables $D_{t}, t=$ 
Table 2 Impact of the maximum acceptable risk level $\epsilon$

\begin{tabular}{|c|c|c|c|c|c|c|c|c|}
\hline$\overline{D_{1}}$ & $\bar{\epsilon}$ & Formulation & Bin & Cons & Cost & Prob & Feas & Time \\
\hline \multirow[t]{20}{*}{ Uniform } & \multirow[t]{4}{*}{0.15} & $\mathrm{BON}$ & 20 & 40 & 2738.7 & 0.971 & NA & $0.5 \mathrm{~s}$ \\
\hline & & SAExt & 4020 & 6041 & 1987.0 & 0.833 & 1 & $307.6 \mathrm{~s}$ \\
\hline & & PSAExt_U & 1006 & 9056 & 2012.4 & 0.849 & 3 & $2611.8 \mathrm{~s}$ \\
\hline & & PSACons_U & 20 & 6825 & 2057.9 & 0.864 & 9 & $17.4 \mathrm{~s}$ \\
\hline & \multirow[t]{4}{*}{0.10} & $\mathrm{BON}$ & 20 & 40 & 2845.4 & 0.979 & NA & $0.5 \mathrm{~s}$ \\
\hline & & SAExt & 3020 & 4041 & 2128.9 & 0.883 & 0 & $67.5 \mathrm{~s}$ \\
\hline & & PSAExt_U & 599 & 6671 & 2169.2 & 0.900 & 4 & $2535.3 \mathrm{~s}$ \\
\hline & & PSACons_U & 20 & 5373 & 2199.5 & 0.907 & 8 & $9.3 \mathrm{~s}$ \\
\hline & \multirow[t]{4}{*}{0.05} & $\mathrm{BON}$ & 20 & 40 & 3016.5 & 0.989 & NA & $0.5 \mathrm{~s}$ \\
\hline & & SAExt & 2020 & 2041 & 2343.7 & 0.934 & 0 & $17.1 \mathrm{~s}$ \\
\hline & & PSAExt_U & 241 & 4149 & 2395.9 & 0.949 & 5 & $2423.1 \mathrm{~s}$ \\
\hline & & PSACons_U & 20 & 3651 & 2411.0 & 0.951 & 5 & $7.7 \mathrm{~s}$ \\
\hline & \multirow[t]{4}{*}{0.02} & $\mathrm{BON}$ & 20 & 40 & 3221.5 & 0.995 & NA & $0.5 \mathrm{~s}$ \\
\hline & & SAExt & 1420 & 841 & 2556.6 & 0.968 & 0 & $5.1 \mathrm{~s}$ \\
\hline & & PSAExt_U & 76 & 2502 & 2631.5 & 0.977 & 2 & $23.4 \mathrm{~s}$ \\
\hline & & PSACons_U & 20 & 2363 & 2637.6 & 0.978 & 4 & $4.1 \mathrm{~s}$ \\
\hline & \multirow[t]{4}{*}{0.01} & $\mathrm{BON}$ & 20 & 40 & 3363.7 & 0.997 & $\mathrm{NA}$ & $0.5 \mathrm{~s}$ \\
\hline & & SAExt & 1220 & 441 & 2670.6 & 0.977 & 0 & $2.6 \mathrm{~s}$ \\
\hline & & PSAExt_U & 41 & 1901 & 2772.3 & 0.987 & 0 & $14.2 \mathrm{~s}$ \\
\hline & & PSACons_U & 20 & 1839 & 2776.0 & 0.987 & 0 & $2.4 \mathrm{~s}$ \\
\hline \multirow[t]{15}{*}{ Normal } & \multirow[t]{3}{*}{0.15} & $\mathrm{BON}$ & 20 & 40 & 2346.1 & 0.966 & $\mathrm{NA}$ & $0.5 \mathrm{~s}$ \\
\hline & & SAExt & 4020 & 6041 & 1817.9 & 0.832 & 0 & $555.2 \mathrm{~s}$ \\
\hline & & PSACons_N & 20 & 47153 & 1931.3 & 0.880 & 10 & $233.0 \mathrm{~s}$ \\
\hline & \multirow[t]{3}{*}{0.10} & $\mathrm{BON}$ & 20 & 40 & 2437.2 & 0.976 & NA & $0.5 \mathrm{~s}$ \\
\hline & & SAExt & 3020 & 4041 & 1951.4 & 0.882 & 0 & $137.9 \mathrm{~s}$ \\
\hline & & PSACons_N & 20 & 17959 & 2068.8 & 0.920 & 10 & $53.6 \mathrm{~s}$ \\
\hline & \multirow[t]{3}{*}{0.05} & $\mathrm{BON}$ & 20 & 40 & 2584.1 & 0.987 & NA & $0.5 \mathrm{~s}$ \\
\hline & & SAExt & 2020 & 2041 & 2140.8 & 0.934 & 0 & $20.3 \mathrm{~s}$ \\
\hline & & PSACons_N & 20 & 10362 & 2265.8 & 0.958 & 10 & $25.1 \mathrm{~s}$ \\
\hline & \multirow[t]{3}{*}{0.02} & $\mathrm{BON}$ & 20 & 40 & 2771.2 & 0.994 & NA & $0.5 \mathrm{~s}$ \\
\hline & & SAExt & 1420 & 841 & 2346.5 & 0.964 & 0 & $5.1 \mathrm{~s}$ \\
\hline & & PSACons_N & 20 & 5420 & 2498.0 & 0.982 & 8 & $11.3 \mathrm{~s}$ \\
\hline & \multirow[t]{3}{*}{0.01} & $\mathrm{BON}$ & 20 & 40 & 2897.6 & 0.997 & NA & $0.5 \mathrm{~s}$ \\
\hline & & SAExt & 1220 & 441 & 2492.8 & 0.979 & 0 & $3.3 \mathrm{~s}$ \\
\hline & & PSACons_N & 20 & 3377 & 2658.2 & 0.990 & 5 & $6.3 \mathrm{~s}$ \\
\hline
\end{tabular}

$1 \ldots T$, seems to have a limited impact on both the Bonferroni approximation and the sample approximation approaches.

Second, for the case where $D_{1}$ follows a uniform distribution, we note that formulation PSAExt_U, which is equivalent to problem PSA, fails at providing feasible solutions of problem SLS when there are positive correlations between variables $D_{t}$. This might be explained as follows. When $D_{1}$ is positively correlated with $D_{t}, t=2 \ldots T$, the probability that the cumulative demand $D C$ takes very large values is higher than when there is no correlation. In formulation PSAExt_U, this is not taken into account so that the stockout risk is underestimated. Conversely, formulation PSAExt_U tends to provide more conservative solutions when there are negative correlations between variables $D_{t}$. This is due to the fact that the probability that $D C$ takes very large values is smaller in case of negative correlations than in case of no correlation, so that in formulation PSAExt_U, the stockout risk tends to be overestimated.

As for the conservative approximations of problem PSA, PSACons_U and PSACons_N, the effects described in the previous paragraph are counterbalanced 
Table 3 Impact of the planning horizon length $T$

\begin{tabular}{|c|c|c|c|c|c|c|c|c|}
\hline$D_{1}$ & $\mathrm{~T}$ & Formulation & Bin & Cons & Cost & Prob & Feas & Time \\
\hline \multirow[t]{12}{*}{ Uniform } & \multirow[t]{4}{*}{10} & $\overline{\mathrm{BON}}$ & 10 & 20 & 1074.7 & 0.982 & NA & $0.2 \mathrm{~s}$ \\
\hline & & SAExt & 1510 & 1021 & 942.2 & 0.937 & 0 & $1.8 \mathrm{~s}$ \\
\hline & & PSAExt_U & 61 & 2725 & 962.6 & 0.951 & 7 & $11.0 \mathrm{~s}$ \\
\hline & & PSACons_U & 10 & 2619 & 964.0 & 0.951 & 7 & $2.5 \mathrm{~s}$ \\
\hline & \multirow[t]{4}{*}{20} & $\overline{\mathrm{BON}}$ & 20 & 40 & 3016.5 & 0.989 & $\mathrm{NA}$ & $0.5 \mathrm{~s}$ \\
\hline & & SAExt & 2020 & 2041 & 2343.7 & 0.934 & 0 & $17.1 \mathrm{~s}$ \\
\hline & & PSAExt_U & 241 & 4149 & 2395.9 & 0.949 & 5 & 2423.1s \\
\hline & & PSACons_U & 20 & 3651 & 2411.0 & 0.951 & 5 & $7.7 \mathrm{~s}$ \\
\hline & \multirow[t]{4}{*}{30} & $\mathrm{BON}$ & 30 & 60 & 6303.9 & 0.993 & $\mathrm{NA}$ & $1.1 \mathrm{~s}$ \\
\hline & & SAExt & 2530 & 3061 & 3903.4 & 0.924 & 0 & $222.7 \mathrm{~s}$ \\
\hline & & PSAExt_U & 469 & 5568 & - & - & - & - \\
\hline & & PSACons_U & 30 & 4519 & 4100.4 & 0.950 & 5 & $16.2 \mathrm{~s}$ \\
\hline \multirow[t]{9}{*}{ Uniform } & \multirow[t]{3}{*}{10} & $\mathrm{BON}$ & 10 & 20 & 999.0 & 0.984 & $\mathrm{NA}$ & $0.3 \mathrm{~s}$ \\
\hline & & SAExt & 1510 & 1021 & 882.7 & 0.941 & 0 & $2.4 \mathrm{~s}$ \\
\hline & & PSACons_N & 10 & 6586 & 918.0 & 0.960 & 10 & $10.3 \mathrm{~s}$ \\
\hline & \multirow[t]{3}{*}{20} & $\mathrm{BON}$ & 20 & 40 & 2584.1 & 0.987 & $\mathrm{NA}$ & $0.5 \mathrm{~s}$ \\
\hline & & SAExt & 2020 & 2041 & 2140.8 & 0.934 & 0 & $20.3 \mathrm{~s}$ \\
\hline & & PSACons_N & 20 & 10362 & 2265.8 & 0.958 & 10 & $25.1 \mathrm{~s}$ \\
\hline & \multirow[t]{3}{*}{30} & $\mathrm{BON}$ & 30 & 60 & 4567.9 & 0.988 & $\mathrm{NA}$ & $1.1 \mathrm{~s}$ \\
\hline & & SAExt & 2530 & 3061 & 3659.8 & 0.929 & 0 & 554.1s \\
\hline & & PSACons_N & 30 & 13240 & 3928.5 & 0.959 & 10 & $63.6 \mathrm{~s}$ \\
\hline
\end{tabular}

"- "indicates that no guaranteed optimal solution could be found within one hour of computation.

Table 4 Impact of the ratio $f / h$

\begin{tabular}{|c|c|c|c|c|c|c|c|c|}
\hline$D_{1}$ & $f / h$ & Formulation & Bin & Cons & Cost & Prob & Feas & Time \\
\hline \multirow[t]{12}{*}{ Uniform } & \multirow[t]{4}{*}{25} & $\mathrm{BON}$ & 20 & 40 & 2627.9 & 0.984 & $\mathrm{NA}$ & $0.5 \mathrm{~s}$ \\
\hline & & SAExt & 2020 & 2041 & 2004.6 & 0.936 & 0 & $8.9 \mathrm{~s}$ \\
\hline & & PSAExt_U & 255 & 4138 & 2086.2 & 0.948 & 1 & 1933.1s \\
\hline & & PSACons_U & 20 & 3584 & 2107.9 & 0.951 & 7 & $4.8 \mathrm{~s}$ \\
\hline & \multirow[t]{4}{*}{50} & $\mathrm{BON}$ & 20 & 40 & 3016.5 & 0.989 & $\mathrm{NA}$ & $0.5 \mathrm{~s}$ \\
\hline & & SAExt & 2020 & 2041 & 2343.7 & 0.934 & 0 & $17.1 \mathrm{~s}$ \\
\hline & & PSAExt_U & 241 & 4149 & 2395.9 & 0.949 & 5 & 2423.1s \\
\hline & & PSACons_U & 20 & 3651 & 2411.0 & 0.951 & 5 & $7.7 \mathrm{~s}$ \\
\hline & \multirow[t]{4}{*}{75} & $\mathrm{BON}$ & 20 & 40 & 3266.4 & 0.990 & $\overline{\mathrm{NA}}$ & $0.5 \mathrm{~s}$ \\
\hline & & SAExt & 2020 & 2041 & 2583.2 & 0.936 & 0 & $19.8 \mathrm{~s}$ \\
\hline & & PSAExt_U & 246 & 4166 & 2635.2 & 0.949 & 3 & $1691.2 \mathrm{~s}$ \\
\hline & & PSACons_U & 20 & 3644 & 2656.3 & 0.952 & 7 & $10.4 \mathrm{~s}$ \\
\hline \multirow[t]{9}{*}{ Normal } & \multirow[t]{3}{*}{25} & $\mathrm{BON}$ & 20 & 40 & 2231.2 & 0.983 & $\mathrm{NA}$ & $0.5 \mathrm{~s}$ \\
\hline & & SAExt & 2020 & 2041 & 1815.0 & 0.923 & 0 & $13.9 \mathrm{~s}$ \\
\hline & & PSACons_N & 20 & 10441 & 1941.3 & 0.954 & 8 & $17.0 \mathrm{~s}$ \\
\hline & \multirow[t]{3}{*}{50} & $\overline{\mathrm{BON}}$ & 20 & 40 & 2584.1 & 0.987 & $\mathrm{NA}$ & $0.5 \mathrm{~s}$ \\
\hline & & SAExt & 2020 & 2041 & 2140.8 & 0.934 & 0 & $20.3 \mathrm{~s}$ \\
\hline & & PSACons_N & 20 & 10362 & 2265.8 & 0.958 & 10 & $25.1 \mathrm{~s}$ \\
\hline & \multirow[t]{3}{*}{75} & $\mathrm{BON}$ & 20 & 40 & 2834.1 & 0.986 & $\mathrm{NA}$ & $0.5 \mathrm{~s}$ \\
\hline & & SAExt & 2020 & 2041 & 2396.5 & 0.933 & 0 & $20.9 \mathrm{~s}$ \\
\hline & & PSACons_N & 20 & 10208 & 2519.1 & 0.958 & 10 & $35.3 \mathrm{~s}$ \\
\hline
\end{tabular}

by the fact that the approximation tends to be more (resp. less) conservative when there are positive (resp. negative) correlations. Namely, in the presence of positive (resp. negative) correlations, the number of scenarios for which the value of the probability $\tilde{\pi}_{i}$ is negative, i.e. is underestimated, is larger (resp. smaller) than the number of such scenarios in the absence of correlations. 
Table 5 Impact of the capacity $c$

\begin{tabular}{|c|c|c|c|c|c|c|c|c|}
\hline$\overline{D_{1}}$ & $\bar{c}$ & Formulation & Bin & Cons & Cost & Prob & Feas & Time \\
\hline \multirow{12}{*}{ Uniform } & \multirow[t]{4}{*}{50} & $\mathrm{BON}$ & 20 & 40 & 3127.9 & 0.985 & $\mathrm{NA}$ & $0.5 \mathrm{~s}$ \\
\hline & & SAExt & 2020 & 2041 & 2451.7 & 0.933 & 0 & $9.7 \mathrm{~s}$ \\
\hline & & PSAExt_U & 255 & 4152 & 2510.6 & 0.949 & 3 & $2385.9 \mathrm{~s}$ \\
\hline & & PSACons_U & 20 & 3007 & 2534.6 & 0.953 & 7 & $5.1 \mathrm{~s}$ \\
\hline & \multirow[t]{4}{*}{100} & $\mathrm{BON}$ & 20 & 40 & 3016.5 & 0.989 & $\mathrm{NA}$ & $0.5 \mathrm{~s}$ \\
\hline & & SAExt & 2020 & 2041 & 2343.7 & 0.934 & 0 & $17.1 \mathrm{~s}$ \\
\hline & & PSAExt_U & 241 & 4149 & 2395.9 & 0.949 & 5 & $2423.1 \mathrm{~s}$ \\
\hline & & PSACons_U & 20 & 3651 & 2411.0 & 0.951 & 5 & $7.7 \mathrm{~s}$ \\
\hline & \multirow[t]{4}{*}{150} & $\overline{\mathrm{BON}}$ & 20 & 40 & 3016.5 & 0.989 & $\mathrm{NA}$ & $0.5 \mathrm{~s}$ \\
\hline & & SAExt & 2020 & 2041 & 2322.5 & 0.932 & 0 & $22.0 \mathrm{~s}$ \\
\hline & & PSAExt_U & 247 & 4107 & 2378.5 & 0.946 & 2 & $714.5 \mathrm{~s}$ \\
\hline & & PSACons_U & 20 & 3636 & 2398.5 & 0.950 & 5 & $8.4 \mathrm{~s}$ \\
\hline \multirow[t]{9}{*}{ Normal } & \multirow[t]{3}{*}{50} & $\mathrm{BON}$ & 20 & 40 & Unf. & NA & $\overline{\mathrm{NA}}$ & $\mathrm{NA}$ \\
\hline & & SAExt & 2020 & 2041 & 2364.8 & 0.931 & 1 & $4.8 \mathrm{~s}$ \\
\hline & & PSACons_N & 20 & 10304 & 2794.6 & 0.969 & 9 & $7.4 \mathrm{~s}$ \\
\hline & \multirow[t]{3}{*}{100} & $\mathrm{BON}$ & 20 & 40 & 2584.1 & 0.987 & $\mathrm{NA}$ & $0.5 \mathrm{~s}$ \\
\hline & & SAExt & 2020 & 2041 & 2140.8 & 0.934 & 0 & $20.3 \mathrm{~s}$ \\
\hline & & PSACons_N & 20 & 10362 & 2265.8 & 0.958 & 10 & $25.1 \mathrm{~s}$ \\
\hline & \multirow[t]{3}{*}{150} & $\mathrm{BON}$ & 20 & 40 & 2584.1 & 0.987 & $\mathrm{NA}$ & $0.5 \mathrm{~s}$ \\
\hline & & SAExt & 2020 & 2041 & 2148.4 & 0.933 & 0 & $31.5 \mathrm{~s}$ \\
\hline & & PSACons_N & 20 & 10224 & 2266.1 & 0.958 & 10 & $20.6 \mathrm{~s}$ \\
\hline
\end{tabular}

"Unf." stands for 'unfeasible'.

Table 6 Impact of the standard deviation

\begin{tabular}{|c|c|c|c|c|c|c|c|c|}
\hline$\overline{D_{1}}$ & {$\left[L_{1}, U_{1}\right]$} & Formulation & Bin & Cons & Cost & Prob & Feas & Time \\
\hline \multirow[t]{12}{*}{ Uniform } & \multirow[t]{4}{*}[20;40]{} & $\overline{\mathrm{BON}}$ & 20 & 40 & 1908.2 & 0.989 & $\mathrm{NA}$ & $0.5 \mathrm{~s}$ \\
\hline & & SAExt & 2020 & 2041 & 1560.6 & 0.932 & 0 & $9.8 \mathrm{~s}$ \\
\hline & & PSAExt_U & 251 & 4151 & 1591.6 & 0.969 & 4 & $1015.1 \mathrm{~s}$ \\
\hline & & PSACons_U & 20 & 3651 & 1601.7 & 0.952 & 8 & $6.5 \mathrm{~s}$ \\
\hline & \multirow{4}{*}[10;50]{} & $\mathrm{BON}$ & 20 & 40 & 3016.5 & 0.989 & $\mathrm{NA}$ & $0.5 \mathrm{~s}$ \\
\hline & & SAExt & 2020 & 2041 & 2343.7 & 0.934 & 0 & $17.1 \mathrm{~s}$ \\
\hline & & PSAExt_U & 241 & 4149 & 2395.9 & 0.949 & 5 & $2423.1 \mathrm{~s}$ \\
\hline & & PSACons_U & 20 & 3651 & 2411.0 & 0.951 & 5 & $7.7 \mathrm{~s}$ \\
\hline & \multirow[t]{4}{*}[0;60]{} & $\mathrm{BON}$ & 20 & $\overline{40}$ & 4118.7 & 0.988 & $\mathrm{NA}$ & $0.5 \mathrm{~s}$ \\
\hline & & SAExt & 2020 & 2041 & 3088.2 & 0.933 & 0 & $9.5 \mathrm{~s}$ \\
\hline & & PSAExt_U & 244 & 4149 & 3171.9 & 0.949 & 6 & $1353.0 \mathrm{~s}$ \\
\hline & & PSACons_U & 20 & 2633 & 3204.4 & 0.952 & 8 & $6.0 \mathrm{~s}$ \\
\hline$D_{1}$ & $s_{1}$ & Formulation & Bin & Cons & Cost & Prob & Feas & Time \\
\hline \multirow[t]{9}{*}{ Normal } & \multirow[t]{3}{*}{5} & $\overline{\mathrm{BON}}$ & 20 & 40 & 1692.1 & 0.987 & $\mathrm{NA}$ & $0.5 \mathrm{~s}$ \\
\hline & & SAExt & 2020 & 2041 & 1469.1 & 0.934 & 0 & $9.1 \mathrm{~s}$ \\
\hline & & PSACons_N & 20 & 10456 & 1529.4 & 0.958 & 10 & $21.1 \mathrm{~s}$ \\
\hline & \multirow[t]{3}{*}{10} & $\overline{\mathrm{BON}}$ & 20 & 40 & 2584.1 & 0.987 & NA & $0.5 \mathrm{~s}$ \\
\hline & & SAExt & 2020 & 2041 & 2140.8 & 0.934 & 0 & $20.3 \mathrm{~s}$ \\
\hline & & PSACons_N & 20 & 10362 & 2265.8 & 0.958 & 10 & $25.1 \mathrm{~s}$ \\
\hline & \multirow[t]{3}{*}{15} & $\overline{\mathrm{BON}}$ & 20 & 40 & 3478.1 & 0.986 & $\mathrm{NA}$ & $0.5 \mathrm{~s}$ \\
\hline & & SAExt & 2020 & 2041 & 2733.3 & 0.918 & 0 & $8.6 \mathrm{~s}$ \\
\hline & & PSACons_N & 20 & 10459 & 2958.2 & 0.952 & 1 & $22.2 \mathrm{~s}$ \\
\hline
\end{tabular}


Table 7 Impact of correlations between $D_{1}$ and $D_{2}, \ldots, D_{t}$

\begin{tabular}{|c|c|c|c|c|c|c|c|c|}
\hline$r(t)$ & $\rho$ & Formulation & Bin & Cons & Cost & Prob & Feas & Time \\
\hline \multirow[t]{20}{*}{ Uniform } & \multirow[t]{4}{*}{1} & $\overline{\mathrm{BON}}$ & 20 & 40 & 10219.9 & 0.983 & $\mathrm{NA}$ & $0.7 \mathrm{~s}$ \\
\hline & & SAExt & 2020 & 2041 & 8521.5 & 0.931 & 0 & $3.5 \mathrm{~s}$ \\
\hline & & PSAExt_U & 740 & 3883 & 8521.4 & 0.933 & 0 & $1825.7 \mathrm{~s}$ \\
\hline & & PSACons_U & 20 & 2130 & 9158.4 & 0.955 & 8 & $1.2 \mathrm{~s}$ \\
\hline & \multirow[t]{4}{*}{0.5} & $\overline{\mathrm{BON}}$ & 20 & 40 & 4385.9 & 0.984 & $\overline{\mathrm{NA}}$ & $0.6 \mathrm{~s}$ \\
\hline & & SAExt & 2020 & 2041 & 3471.9 & 0.933 & 0 & $11.9 \mathrm{~s}$ \\
\hline & & PSAExt_U & 474 & 3883 & 3489.9 & 0.937 & 0 & $2054.5 \mathrm{~s}$ \\
\hline & & PSACons_U & 20 & 2761 & 3642.4 & 0.950 & 6 & $3.2 \mathrm{~s}$ \\
\hline & \multirow[t]{4}{*}{0.0} & $\overline{\mathrm{BON}}$ & 20 & 40 & 3016.5 & 0.989 & $\mathrm{NA}$ & $0.5 \mathrm{~s}$ \\
\hline & & SAExt & 2020 & 2041 & 2343.7 & 0.934 & 0 & $17.1 \mathrm{~s}$ \\
\hline & & PSAExt_U & 241 & 4149 & 2395.9 & 0.949 & 5 & 2423.1s \\
\hline & & PSACons_U & 0 & 3651 & 2411.0 & 0.951 & 5 & $7.7 \mathrm{~s}$ \\
\hline & \multirow[t]{4}{*}{-0.5} & $\overline{\mathrm{BON}}$ & 20 & 40 & 2183.0 & 0.985 & $\overline{\mathrm{NA}}$ & $0.7 \mathrm{~s}$ \\
\hline & & SAExt & 2020 & 2041 & 1897.6 & 0.935 & 0 & $13.4 \mathrm{~s}$ \\
\hline & & PSAExt_U & 126 & 4491 & 2003.9 & 0.959 & 10 & $108.5 \mathrm{~s}$ \\
\hline & & PSACons_U & 20 & 4253 & 2007.6 & 0.960 & 10 & $8.8 \mathrm{~s}$ \\
\hline & \multirow[t]{4}{*}{-1} & $\mathrm{BON}$ & 20 & 40 & 2161.9 & 0.986 & $\mathrm{NA}$ & $0.7 \mathrm{~s}$ \\
\hline & & SAExt & 2020 & 2041 & 1881.7 & 0.933 & 0 & $6.9 \mathrm{~s}$ \\
\hline & & PSAExt_U & 121 & 4512 & 2040.9 & 0.956 & 10 & $43.3 \mathrm{~s}$ \\
\hline & & PSACons_U & 20 & 4282 & 2042.0 & 0.956 & 10 & $8.9 \mathrm{~s}$ \\
\hline \multirow[t]{15}{*}{ Normal } & \multirow[t]{3}{*}{1} & $\overline{\mathrm{BON}}$ & 20 & 40 & 11984.5 & 0.991 & $\mathrm{NA}$ & $0.5 \mathrm{~s}$ \\
\hline & & SAExt & 2020 & 2041 & 9219.7 & 0.939 & 1 & $2.7 \mathrm{~s}$ \\
\hline & & PSACons_N & 20 & 4466 & 10569.3 & 0.970 & 10 & $1.5 \mathrm{~s}$ \\
\hline & \multirow[t]{3}{*}{0.5} & $\overline{\mathrm{BON}}$ & 20 & 40 & 4029.5 & 0.987 & $\mathrm{NA}$ & $0.5 \mathrm{~s}$ \\
\hline & & SAExt & 2020 & 2041 & 3143.9 & 0.934 & 0 & $14.7 \mathrm{~s}$ \\
\hline & & PSACons_N & 20 & 7308 & 3382.7 & 0.957 & 8 & $14.6 \mathrm{~s}$ \\
\hline & \multirow[t]{3}{*}{0.0} & $\overline{\mathrm{BON}}$ & 20 & 40 & 2584.1 & 0.987 & $\mathrm{NA}$ & $0.5 \mathrm{~s}$ \\
\hline & & SAExt & 2020 & 2041 & 2140.8 & 0.934 & 0 & $20.3 \mathrm{~s}$ \\
\hline & & PSACons_N & 20 & 10456 & 1529.4 & 0.958 & 10 & $21.1 \mathrm{~s}$ \\
\hline & \multirow[t]{3}{*}{-0.5} & $\overline{\mathrm{BON}}$ & 20 & 40 & 2052.3 & 0.986 & $\mathrm{NA}$ & $0.5 \mathrm{~s}$ \\
\hline & & SAExt & 2020 & 2041 & 1757.8 & 0.932 & 0 & $18.9 \mathrm{~s}$ \\
\hline & & PSACons_N & 20 & 12642 & 1909.1 & 0.966 & 10 & $48.7 \mathrm{~s}$ \\
\hline & \multirow[t]{3}{*}{1} & $\overline{\mathrm{BON}}$ & 20 & 40 & 2068.8 & 0.987 & $\mathrm{NA}$ & $0.5 \mathrm{~s}$ \\
\hline & & SAExt & 2020 & 2041 & 1770.7 & 0.933 & 0 & $7.6 \mathrm{~s}$ \\
\hline & & PSACons_N & 20 & 12409 & 1941.9 & 0.961 & 10 & $46.8 \mathrm{~s}$ \\
\hline
\end{tabular}

We thus conclude that, in the presence of correlations between $D_{1}$ and $D_{t}, t=$ $2 \ldots T$, formulations PSACons_U and PSACons_N might be useful to find feasible solutions of problem SLS within a reduced computation time. However, these solutions might be overly conservative. Thus, in case a close-to-optimal solution is required, the use of formulation SAExt with a large sample size might be more appropriate.

\subsection{Numerical results: assessment within a rolling horizon framework}

In practice, production planning is often carried out within a rolling horizon framework. In such a framework, the production plan is computed for a planning horizon of $T$ periods but only the decisions pertaining to the rolling horizon window, i.e. to the first $T_{r h}$ periods, are actually implemented. At the end of period $T_{r h}$, a new production plan is computed for the $T$ following periods taking into account 
the realization of the stochastic demand over periods 1 to $T_{r h}$ and the updated probabilistic information on the demand over periods $T_{r h}+1 \ldots T_{r h}+T$.

In this subsection, we thus simulate the implementation of the production plans computed either with the sample approximation or with the partial sample approximation within a rolling horizon framework. We seek to evaluate the actual cost and the actual stockout risk of these approaches when used in a rolling horizon planning environment. Note that the cost considered here is not the objective function of the MILP formulation but the sum over time of the actual costs incurred when applying the production planning decisions on the periods belonging to the rolling horizon window. Similarly, the actual stockout risk is not estimated by computing the value of Prob as done in the previous subsections but by checking whether applying the production planning decisions on the rolling horizon window with the actual value of the demand leads to a stockout.

More precisely, we use the rolling horizon simulation procedure described as Algorithm 1. Running the simulation once means solving an MILP several times. Moreover, in order to get a good statistical evaluation of the true cost and stockout risk, we run the simulation 500 times. In view of this significant computational effort, we focus in our experiments on the reference data set in which $T=20$, $h=1, f=50, c=100, N=1000, \epsilon=0.05$ and the demands $D_{t}$ are independent of one another and follow a uniform distribution on $[10,50]$. We considered three values of the rolling horizon window: $T_{r h} \in\{5,10,15\}$. We also considered the value $T_{r h}=20$ in order to simulate a static planning environment.

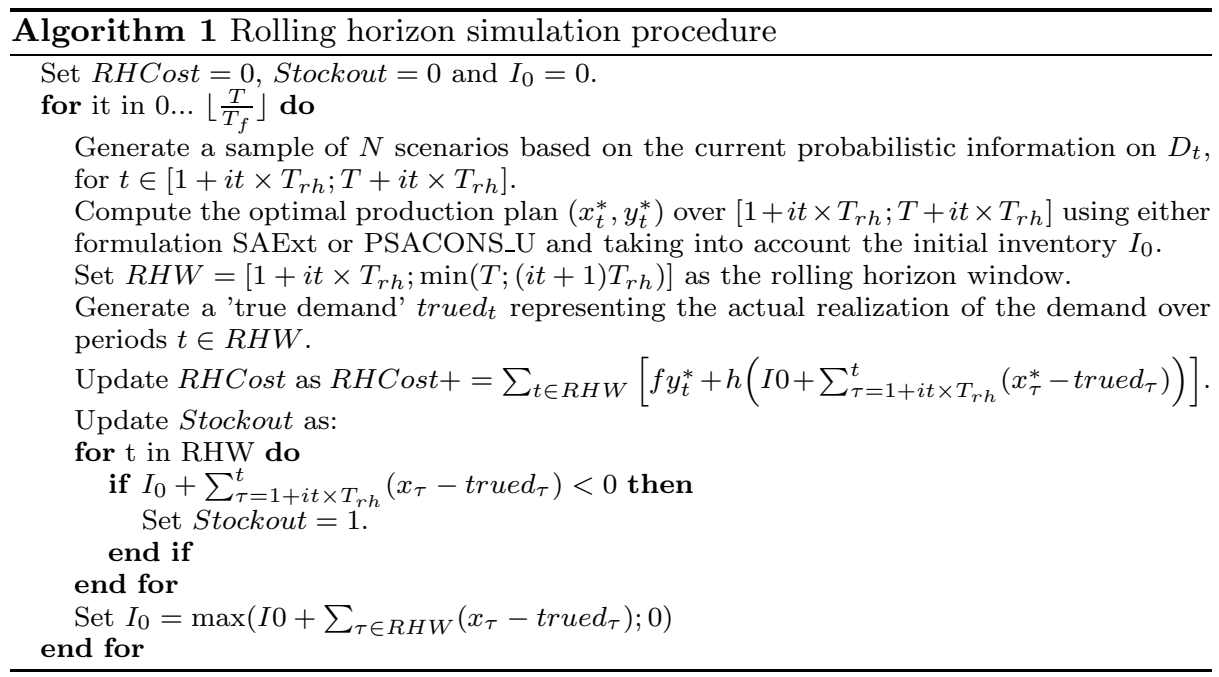

The corresponding numerical results are displayed in Table 8. For each value of $T_{r h}$, we provide the average value of $R H C$ Cost over the 500 simulation replications and RHProb the percentage of replications in which there is no stockout during the planning horizon.

Results from Table 8 first show that, in a rolling horizon framework, the actual cost is lower than the cost of the optimal solution obtained with a static planning horizon. Moreover, the shorter the rolling horizon window, the larger the cost 
Table 8 Assessment within a rolling horizon framework

\begin{tabular}{|c|c|c|cc|}
\hline Demand & $T_{r h}$ & Formulation & RHCost & RHProb \\
\hline Uniform & 5 & SAExt & 1833.1 & 0.968 \\
& & PSACons_U & 1902.8 & 0.986 \\
\cline { 2 - 5 } & 10 & SAExt & 2055.0 & 0.942 \\
& & PSACons_U & 2093.6 & 0.966 \\
\cline { 2 - 5 } & 15 & SAExt & 2145.6 & 0.960 \\
& & PSACons_U & 2221.2 & 0.966 \\
\cline { 2 - 5 } & 20 & SAExt & 2350.1 & 0.948 \\
& & PSACons_U & 2434.1 & 0.968 \\
\hline
\end{tabular}

reduction. This might be explained as follows. During iteration it of the rolling horizon planning procedure, the inventory level at the end of period $(i t+1) T_{r h}$, which is computed based on the actual realization of the demand over the rolling horizon window, becomes the initial inventory for the iteration it +1 . When this initial inventory is strictly positive, it is possible to use it in the resolution of the MILP formulations SAExt or PSACons_U in order to reduce the production quantities while ensuring the same service level over the next $T$ periods. The shorter the rolling horizon window, the more frequently the initial inventory can be updated and the more frequently the production plan can be readjusted to take it into account.

Regarding the actual stockout risk, we can see from the results in Table 8 that it tends to be lower in a rolling horizon framework than in a static planning horizon. Note however that this conclusion should be taken carefully as the limited number of replications (500) in our simulation may not enable us to get a good statistical evaluation of the stockout risk, especially as stockouts occur only for very large and statistically infrequent values of the cumulative demand. A possible explanation for this decrease in the stockout risk might be that in the solutions obtained by formulations SAExt or PSACons_U, the stockout risk is much higher in the last periods of the horizon than in the first periods. For example, let us consider the optimal solutions of formulation SAExt for the 10 instances of the reference data set. For each instance, we focus on the $\lfloor N \epsilon\rfloor=50$ scenarios displaying a stockout (i.e. such that $\alpha_{i}^{*}=1$ ). We note that only $21 \%$ of the stockouts are planned to occur during periods 1 to 10 and $79 \%$ are planned to occur during periods 10 to 20 . As, in a rolling horizon framework, only the first periods of the production plan are implemented before readjusting the plan, the actual stockout risk observed in a rolling horizon framework may be smaller than the one observed in a static planning environment.

\section{Conclusion and perspectives}

We studied the single-item single-resource capacitated lot-sizing problem with stochastic demand and proposed to handle this problem using a single-stage stochastic programming approach. More precisely, we formulated this stochastic problem as a joint chance-constrained program where the probability that an inventory shortage occurs during the planning horizon is limited to a maximum acceptable risk level. 
As the resulting probabilistic mixed-integer program is computationally difficult to handle, we investigated the development of an approximate solution method which can be seen as an extension of the previously published sample approximation approach. This extension is based on the assumption that the demand in the first period is statistically independent of the demand in the other periods. Similarly to the the sample approximation approach presented by Luedtke et al [15], the proposed extension relies on a Monte Carlo sampling method. However, this sampling is carried out on only part of the random variables, more precisely on all random variables except $D_{1}$. Provided there is no correlation between $D_{1}$ and the demand in the later periods, this partial sampling results in the formulation of a chance-constrained program featuring a series of joint chance constraints. Each of these constraints involves a single random variable and defines a feasible set for which a conservative convex approximation can be quite easily built. The main advantage of the proposed partial sample approximation approach lies in the fact that it leads to the formulation of a deterministic mixed-integer linear problem having the same number of binary variables as the original problem. Our computational results show that the proposed solution method is more efficient at finding feasible solutions of the original stochastic problem than the sample approximation method and that these solutions are less costly than the ones provided by the Bonferroni conservative approximation. Moreover, the computation time is significantly shorter than the one needed for the sample approximation method.

Among the possible directions for future research suggested by the present work, it might be worth investigating whether the partial sample approximation approach might be extended to solve a joint chance-constraint lot-sizing problem in a dynamic (multi-stage) decision making context such as the one considered by Zhang et al [26]. Moreover, in many industrial cases, the available production resource is not dedicated to the production of a single product but rather shared by several various products. Another possible extension of the present work which could help close the gap between the current state of the art and the industrial need would thus be to consider the multi-item capacitated lot-sizing problem with stochastic demand.

Acknowledgements The work described in the present paper was partly funded by the French National Research Agency through its research funding program for young researchers (Project ANR-11-JS0002-01 LotRelax). The authors would also like to thank two anonymous referees for their detailed reviews that helped improving an initial version of this paper.

\section{Appendix 1: Proof of Proposition 1}

We first note that constraints (50) can be written as:

$$
\pi^{i}=\operatorname{Pr}\left(\min _{t=1 \ldots T}\left(\sum_{\tau=1}^{t} x_{\tau}-\Delta C_{t}^{i}\right) \geq D_{1}\right)=F_{D_{1}}\left(\min _{t=1 \ldots T}\left(\sum_{\tau=1}^{t} x_{\tau}-\Delta C_{t}^{i}\right)\right)
$$

which implies that:

$$
\pi^{i} \leq F_{D_{1}}\left(\sum_{\tau=1}^{t} x_{\tau}-\Delta C_{t}^{i}\right), \forall t=1 \ldots T
$$


The proof of Proposition 1 is done by contradiction.

- for $t=1$

Assume that we have $x_{1}<F_{D_{1}}^{-1}(1-\epsilon)$. This implies that:

$\forall i=1 \ldots N, \pi_{i} \leq F_{D_{1}}\left(x_{1}\right)<F_{D_{1}}\left(F_{D_{1}}^{-1}(1-\epsilon)\right)<1-\epsilon$.

We thus have: $\sum_{i} \pi_{i}<N(1-\epsilon)$. Hence the corresponding production plan violates constraint (51).

This means that any feasible solution of problem PSAConsDet satisfies constraint (97).

- for $t \in[2 ; T]$

Assume that $\sum_{\tau=1}^{t} x_{\tau}<F_{D_{1}}^{-1}(1-\epsilon)+\Delta C_{t}^{\nu_{t}^{k_{t}}}$. We have:

$$
\begin{aligned}
\sum_{i=1}^{N} \pi^{i} & \leq \sum_{i=1}^{N} F_{D_{1}}\left(\sum_{\tau=1}^{t} x_{\tau}-\Delta C_{t}^{i}\right) \\
& \leq \sum_{i=1}^{N} F_{D_{1}}\left(F_{D_{1}}^{-1}(1-\epsilon)+\Delta C_{t}^{\nu_{t}^{k_{t}}}-\Delta C_{t}^{i}\right) \\
& <N(1-\epsilon)
\end{aligned}
$$

Inequality (102) is obtained by using constraints (101) for period $t$. (103) makes use of the assumption mentioned above. By definition of $k_{t}$, the left hand side of inequality (103) is strictly less that $N(1-\epsilon)$, which gives (104). Thus, the corresponding production plan violates constraint (51).

This implies that any feasible solution of problem PSA satisfies constraint (99).

\section{References}

1. Beraldi P. and Ruszczyǹski A., A branch and bound method for stochastic integer problems under probabilistic constraints, Optimization Methods and Software, 17(4), 359-382, 2002.

2. Bonferroni C.E., Teoria statistica delle classi e calcolo delle probabilita. Pubblicazioni del $R$ Istituto Superiore di Scienze Economiche e Commerciali di Firenze, 8, 3-62, 1936.

3. Bookbinder J.H. and Tan J.Y, Strategies for the probabilistic lot-sizing problem with service-level constraints, Management Science, 34(9), 1096-1108, 1988.

4. Buschkühl L., Sahling F., Helber S. and Tempelmeier H., Dynamic capacitated lot-sizing problems: classification and review of solution approaches, OR Spectrum, 32, 231-261, 2010.

5. Calafiore G. and Campi M.C., Uncertain convex programs: randomized solutions and confidence levels, Mathematical Programming, 102, 25-46, 2005.

6. Chen H., A Lagrangian Relaxation approach for production planning with demand uncertainty, European Journal of Industrial Engineering, 1(4), 370-390, 2007.

7. Cheng J., Lisser A. and Gicquel C., A new partial sample average approximation method for chance-constrained problems. Working paper, available at http://www.optimization online.org $/ D B_{H} T M L / 2014 / 11 / 4622 . h t m l, 2014$.

8. Di Summa M. and Wolsey L.A., Lot-sizing on a tree, Operations Research Letters, 36, 7-13, 2008.

9. Guan Y., Ahmed S., Miller A.J. and Nemhauser G.L., On formulations of the stochastic uncapacitated lot-sizing problem, Operations Research Letters, 34, 241-250, 2006.

10. Haugen K.K., Lokketangen A. and Woodruff D.L., Progressive hedging as a metaheuristic applied to stochastic lot-sizing, European Journal of Operational Research, 132, 116-122, 2001. 
11. Jans R. and Degraeve Z., Modeling industrial lot sizing problems: a review. Industrial Journal of Production Research, 46(6), 1619-1643, 2008.

12. Küçükyavuz S., On mixing sets arising in chance-constrained programming, Mathematical Programming, 132, 31-56, 2012.

13. Leung J., Magnanti T.L. and. Vachani R., Facets and algorithms for capacitated lot sizing, Mathematical Programming, 45, 331-359, 1989.

14. Luedtke J. and Ahmed S., A sample approximation approach for optimization with probabilistic constraints, SIAM Journal of Optimization, 19(2), 674-699, 2008.

15. Luedtke J., Ahmed S. and Nemhauser G.L., An integer programming approach for linear programs with probabilistic constraints, Mathematical Programming, 122, 247-272, 2010.

16. Pochet Y. and Wolsey L.A., Production planning by mixed integer programming, Springer Science, 2006.

17. Rockafellar R. T. and Uryasev S. P., Optimization of conditional value-at-risk, Journal of Risk, 2, 21-41, 2000.

18. Nemirovski A. and Shapiro A., Scenario approximations of chance constraints, Probabilistic and Randomized Methods for Design Under Uncertainty, G. Calafiore and F. Dabbene, eds., Springer, London, 3-48, 2005.

19. Nemirovski A. and Shapiro A., Convex approximations of chance constrained programs, SIAM Journal of Optimization, 17, 969-996, 2006.

20. Piperagkas G.S., Konstantaras I., Skouri K. and Parsopoulo K.E., Solving the stochastic dynamic lot-sizing problem through nature-inspired heuristics, Computers E Operations Research, 39, 1555-1565, 2012.

21. Tarim S.A. and Kingsman B.G., The stochastic dynamic production/inventory lot-sizing problem with service-level constraints, International Journal of Production Economics, 88, 105-119, 2004.

22. Tempelmeier H., On the stochastic uncapacitated dynamic single-item lotsizing problem with service level constraints, European Journal of Operational Research, 181, 184-194, 2007.

23. Tempelmeier H. and Herpers S., Dynamic uncapacitated lot sizing with random demand under a fillrate constraint, European Journal of Operational Research, 212, 497-507, 2011.

24. Vargas V., An optimal solution for the stochastic version of the Wagner-Whitin dynamic lot-size model, European Journal of Operational Research, 198, 447-451, 2009.

25. Wagner H.M. and Whitin T.M., Dynamic version of the economic lot size model, Management Science, 5(1), 89-96, 1958.

26. Zhang M., Küçükyavuz S. and Goel S., A branch and cut method for dynamic decision making under joint chance constraints. Management Science, 60(5), 1317-1333, 2014. 